\title{
ARID1A prevents squamous cell carcinoma initiation and chemoresistance by antagonizing pRb/E2F1/c-Myc-mediated cancer stemness
}

\author{
Qingyu Luo $\mathbb{D}^{1} \cdot$ Xiaowei $\mathrm{Wu}^{1} \cdot$ Wan $\mathrm{Chang}^{1} \cdot$ Pengfei Zhao ${ }^{1} \cdot$ Yabing Nan ${ }^{1} \cdot$ Xiaolin Zhu ${ }^{1} \cdot$ Jonathan P. Katz $\mathbb{D}^{2} \cdot$ \\ Dan $\mathrm{Su}^{3} \cdot$ Zhihua Liu $\mathbb{1}^{1}$
}

Received: 2 September 2019 / Revised: 3 December 2019 / Accepted: 3 December 2019 / Published online: 12 December 2019

(c) The Author(s), under exclusive licence to ADMC Associazione Differenziamento e Morte Cellulare 2019

\begin{abstract}
Squamous cell carcinoma (SCC) is defined as a category of aggressive malignancies arising from the squamous epithelium of various organs. Resistance to chemotherapies is a common feature of SCCs, which leads to a poor prognosis among SCC patients. Recently, studies have illustrated the essential tumor suppressive role of ARID1A in several cancer types, but its role in SCCs remains unclear. Cancer stemness has been recognized as a main reason for tumorigenesis and is commonly correlated with chemoresistance, yet the relationship between ARID1A and cancer stemness remains unknown. In this study, we showed that Aridla conditional knockout mice had a high incidence of SCCs occurring in the tongue and esophagus. ARID1A depletion promoted tumor initiation and cancer stemness in human SCC cells. Mechanistic studies revealed that ARID1A blocked the interaction between cyclin-dependent kinases (CDKs) and retinoblastoma protein $(\mathrm{Rb})$, reducing the phosphorylation of $\mathrm{Rb}$. Dephosphorylated Rb suppressed E2F1 activity and then suppressed cancer stemness by inactivating c-Myc. Furthermore, we showed that ARID1A depletion significantly increased the chemoresistance of SCC and that a CDK inhibitor exhibited a favorable effect on rescuing the chemoresistance caused by ARID1A loss. Collectively, our study showed that ARID1A inhibits the cancer stemness of SCCs by competing with CDKs to bind with Rb to inhibit the E2F1/cMyc pathway.
\end{abstract}

These authors contributed equally: Qingyu Luo, Xiaowei Wu

Edited by T. Mak

Supplementary information The online version of this article (https:// doi.org/10.1038/s41418-019-0475-6) contains supplementary material, which is available to authorized users.

Zhihua Liu

liuzh@cicams.ac.cn

1 State Key Laboratory of Molecular Oncology, National Cancer Center/National Clinical Research Center for Cancer/Cancer Hospital, Chinese Academy of Medical Sciences and Peking Union Medical College, Beijing 100021, China

2 Gastroenterology Division, Department of Medicine, University of Pennsylvania, Philadelphia, PA 19104, USA

3 Department of Pathology, Zhejiang Cancer Hospital, Zhejiang 310022, China

\section{Introduction}

Squamous cell carcinoma (SCC) includes a collection of tumors originating from diverse anatomical locations and is one of the most common solid cancers in humans [1]. SCCs usually express squamous differentiation markers, share common genetic mutations, and are classified according to their origins [2]. The most frequently observed locations of SCCs include the skin, head and neck, esophagus, lung, and cervix [2]. Cutaneous squamous cell carcinoma (cSCC) is a nonmelanoma skin cancer type that represents the second most common type $(\sim 20 \%)$ of all skin malignancies, with up to 700,000 new cases diagnosed in the United States per year [3]. Lung squamous cell carcinomas (LSCCs) account for 25-30\% of all lung malignancies and are the leading cause of cancer-related death worldwide [4]. Esophageal carcinoma is the eighth most common cancer and the sixth most common cause of cancer-related deaths worldwide [5, 6], causing more than 400,000 patient deaths each year [5]. Esophageal squamous cell carcinoma (ESCC) accounts for $90 \%$ of all esophageal cancer cases, and the 5-year overall survival of 
patients with ESCC is less than 15\% [7]. SCC patients suffer from a poor prognosis mostly due to resistance to chemotherapies and relapse [8-10], yet the specific mechanism remains not fully understood. For these reasons, there is a clear need to better understand the molecular events that contribute to the poor prognosis of SCC patients and to develop more promising strategies for addressing chemoresistance.

ARID1A plays an essential tumor suppressive role in various cancer types, such as ovarian cancer [11, 12], gastric cancer [13, 14], and colorectal cancer [15, 16]. Moreover, studies of synthetic targeting strategies for ARID1A loss have developed rapidly and revealed several promising clinical applications [17-19]. Nevertheless, existing studies of ARID1A have focused on its functions and mechanisms in adenocarcinomas, yet the role of ARID1A in SCC initiation and progression remains completely unknown. More importantly, if ARID1A, a well-known tumor suppressor, also exerts an essential function in SCCs, uncovering its functions and mechanism will be of great significance for identifying potential strategies to overcome the chemoresistance and poor prognosis of SCC patients.

Here, we revealed for the first time that ARID1A prevents SCC initiation and progression in both murine and human contexts. We further showed that ARID1A loss contributes to tumorigenesis and chemoresistance via promoting cancer stemness. Mechanistically, ARID1A prevents the interaction between cyclin-dependent kinases (CDKs) and retinoblastoma protein $(\mathrm{Rb})$, resulting in the dephosphorylation of $\mathrm{Rb}$, and then prevents $\mathrm{E} 2 \mathrm{~F} 1$ from transcriptionally activating c-Myc. Moreover, we showed that a CDK inhibitor (CDKi) could resensitize ARID1Adepleted SCC cells to cisplatin (DDP) treatment, thus showing the promising potential of a CDKi for treating SCC patients with low ARID1A expression.

\section{Materials and methods}

\section{Cell lines and cell culture}

Human ESCC cell lines [20] were provided by Dr Yutaka Shimada (Kyoto University, Japan). NE-2 and NE-3 cells [21] were gifts from Dr Enmin Li (Medical College of Shantou University, China). The Het-1A and 293T cell lines were purchased from the American Type Culture Collection (ATCC, VA, USA). A431, NCI-H1703 and CAL-27 cells were purchased from the National Infrastructure of Cell Line Resource (Beijing, China). All SCC cells were cultured in RPMI-1640 medium supplemented with $10 \%$ FBS, except for A431 and CAL-27 cells, which were cultured in DMEM supplemented with 10\% FBS. 293T cells were cultured in DMEM supplemented with 10\% FBS. Het-1A cells were cultured in a BEBM Kit (CC-3170, Lonza), and culture dishes were pretreated with an Enhanced Cell Adhesion Kit-3 (ECAK-3) (C028, Beijing Xigong Biological Technology Co., Ltd, Beijing, China) for $1 \mathrm{~h}$. NE-2 and NE-3 cells were cultured in a 1:1 mixture of defined K-SFM (10744-019, Gibco, MA, USA) and EpiLife medium (MEP1500CA, Gibco). All cells were maintained in a humidified cell incubator with $5 \% \mathrm{CO}_{2}$ at $37^{\circ} \mathrm{C}$ and routinely authenticated using short tandem repeat (STR) DNA fingerprinting. Cells were tested by a MycoBlue Mycoplasma Detector (Vazyme Biotech, Nanjing, China) to exclude Mycoplasma contamination before experiments.

\section{Antibodies and reagents}

Antibodies against the following proteins were used for western blotting: ARID1A (1:1000, Cell Signaling Technology; 12354), ABCG2 (1:1000, Cell Signaling Technology; 42078), Nanog (1:2000, Cell Signaling Technology; 4903), Sox2 (1:1000, Cell Signaling Technology; 14962), Keratin 4 (1:5000, Abcam; ab51599), Keratin 13 (1:200,000, Abcam; ab92551), phospho-Rb (1:1000, Cell Signaling Technology; 8516), Rb (1:1000, Cell Signaling Technology; 9309), c-Myc (1:1000, Cell Signaling Technology; 5605), E-Cadherin (1:1000, Cell Signaling Technology; 3195), N-Cadherin (1:1000, Cell Signaling Technology; 13116), ZEB1 (1:1000, Cell Signaling Technology; 3396), and $\beta$-actin (1:5000, Sigma; A5316). The following antibodies were used for chromatin immunoprecipitation (ChIP): E2F1 (1:100, Cell Signaling Technology; 3742) and normal rabbit IgG (Cell Signaling Technology; 2729). The following antibodies were used for immunohistochemistry (IHC): ARID1A (1:500, Sigma; HPA005456), Sox2 (1:200, Cell Signaling Technology; 14962), Ki-67 (1:400, Cell Signaling Technology; 12202), phospho-Rb (1:400, Cell Signaling Technology; 8516), and c-Myc (1:200, Abcam; ab32072). Palbociclib (HY-50767) and anti-Myc magnetic beads (HY-K0206) were purchased from MedChemExpress (NJ, USA).

\section{qRT-PCR and ChIP-qPCR}

Total RNA was isolated from cells using TRIzol reagent (Thermo Scientific, MA, USA). qPCR analysis was performed on a StepOnePlus Real-Time PCR system (Applied Biosystems, CA, USA). ChIP analysis was performed using a ChIP Kit (Cell Signaling Technology, MA, USA) as previously described [22]. All relative expression levels of the target genes were normalized to the housekeeping gene GAPDH. The GAPDH primers were as follows: forward, 5'-CCGGGAAACTGTGGCGTGATGG-3'; reverse, 5'-AG GTGGAGGAGTGGGTGTCGCTGTT- ${ }^{\prime}$. The ARID1A primers were as follows: forward, 5'-CGGCCAATGGATG GCACATA-3'; reverse, 5'-TCGGCCAAACTGGAATGGA A-3'. The c-Myc primers were as follows: forward, 5'-AGG 
A

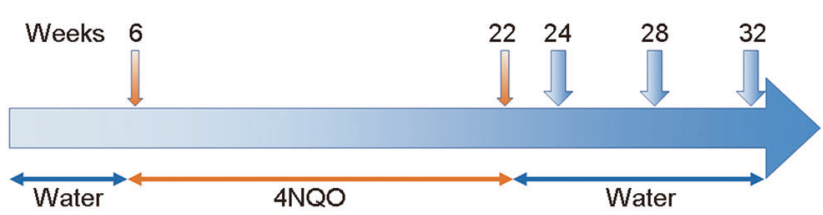

B

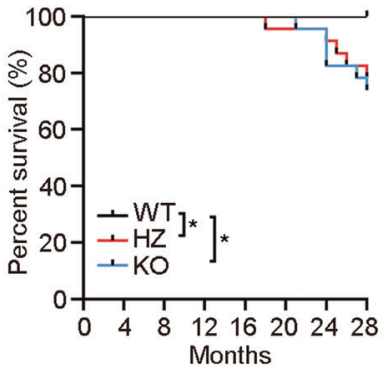

C

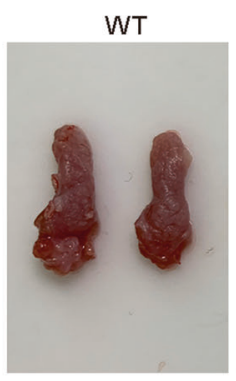

$\mathrm{HZ}$
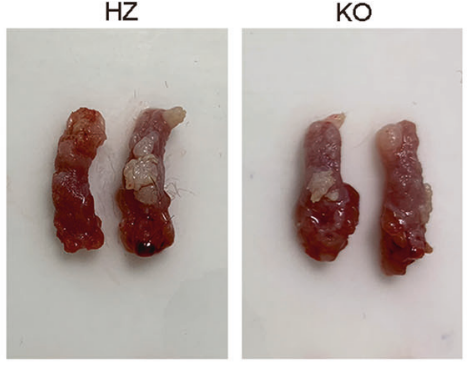

E

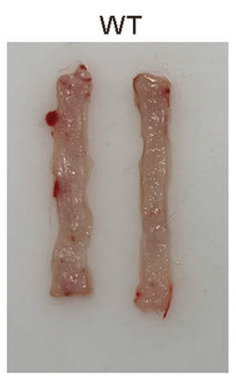

$\mathrm{HZ}$

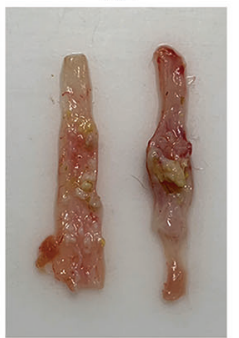

KO

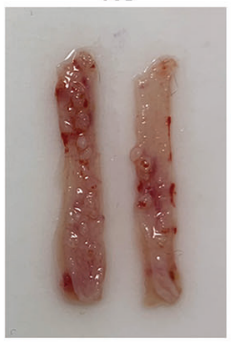

D $\quad 24$ W

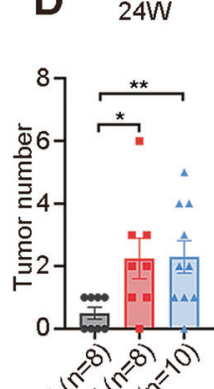

ans

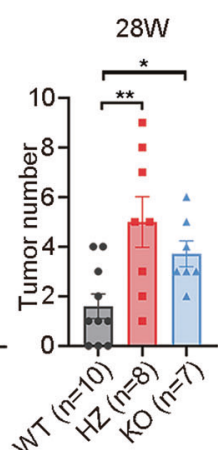

F

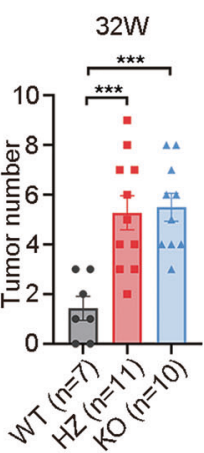

\begin{tabular}{llll}
\hline & WT & HZ & KO \\
\hline $24 \mathrm{~W}$ & $37.5 \%$ & $62.5 \%$ & $70 \%$ \\
& $(3 / 8)$ & $(5 / 8)$ & $(7 / 10)$ \\
$28 \mathrm{~W}$ & $40 \%$ & $62.5 \%$ & $100 \%$ \\
& $(4 / 10)$ & $(5 / 8)$ & $(7 / 7)$ \\
$32 \mathrm{~W}$ & $42.9 \%$ & $100 \%$ & $100 \%$ \\
& $(3 / 7)$ & $(10 / 10)$ & $(11 / 11)$ \\
\hline
\end{tabular}

G WT
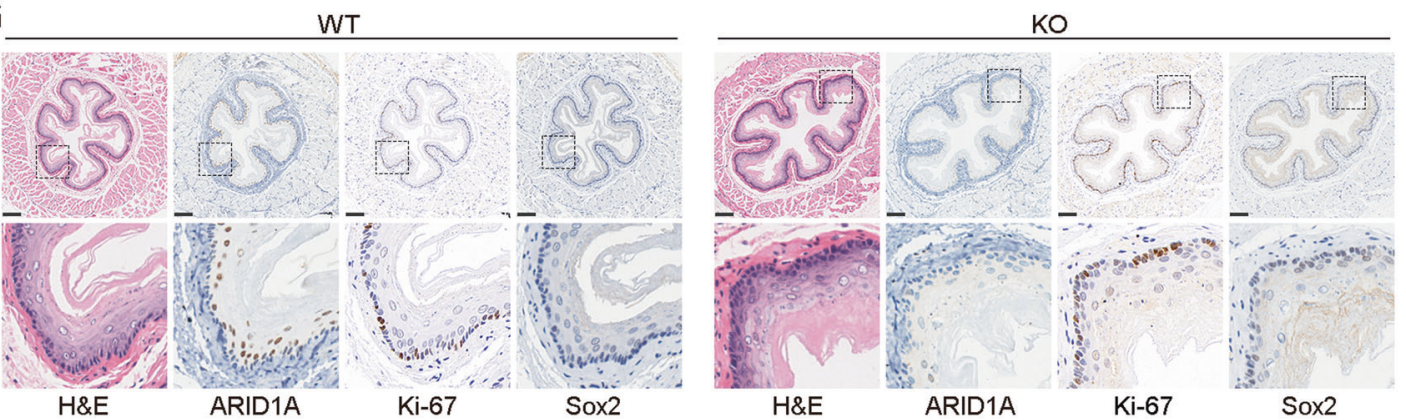

$\mathrm{H}$
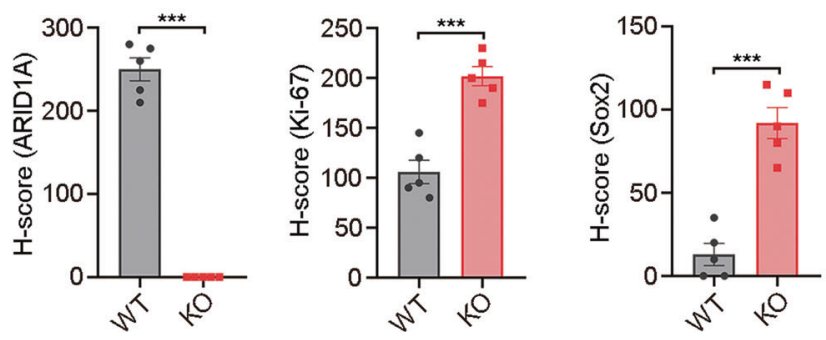

GAGATCCGGAGCGAATA-3'; reverse, 5'-GTCCTTGCT CGGGTGTTGTA-3'. The CDH1 primers were as follows: forward, 5'-TGGATGTGAATGAAGCCCCC-3'; reverse,
5'-AGTGGAAATGGCACCAGTGT-3'. The CDH2 primers were as follows: forward, $5^{\prime}$-GGCTTCTGGTGAAAT CGCAT-3'; reverse, 5'-CTGCCACTTGCCACTTTTCC-3'. 
Fig. 1 Aridla knockout promotes the initiation of tongue and esophageal squamous cell carcinoma in mice. a Schematics showing the schedule of $4 \mathrm{NQO}$ treatment. b Overall survival of mice treated with 4NQO (WT wild-type, $n=17$; HZ heterozygous, $n=23$; KO knockout, $n=23$ ). Representative images (c) and numbers (d) of tongue tumors in 4NQO-induced mice. Representative images (e) and incidence (f) of esophageal tumors in 4NQO-induced mice. $\mathbf{g}$, h Representative images and $\mathrm{H}$-scores of IHC staining for ARID1A, Ki-67, and Sox2 expression in the mouse esophageal epithelium. Scale bars, $100 \mu \mathrm{m}$. Data are shown as the mean \pm s.e.m.; two-tailed $t$ tests; ${ }^{*} P<0.05$, $* * P<0.01$, $* * * P<0.001$.

The ZEB1 primers were as follows: forward, $5^{\prime}$-GATGACC TGCCAACAGACCA-3'; reverse, 5'-GTGTCATCCTCCC AGCAGTT- $3^{\prime}$. The ZEB2 primers were as follows: forward, 5'-CACGATCCAGACCGCAATTA-3'; reverse, 5'-TCAC TGCGCTGAAGGTACTC- $3^{\prime}$. The c-Myc-P1 primers for ChIP-qPCR were as follows: forward, 5'-AGAATAACAA GGAGGTGGCTGGAAAC-3'; reverse, 5'-CTACTGGCAG CAGAGATCATCGC-3'. The c-Myc-P2 primers for ChIPqPCR were as follows: forward, 5'-GGAACAGGCAGA CACATCTCAGG-3'; reverse, 5'-GCACAGCTATCTGGA TTGGATACCTT-3'.

\section{Immunoprecipitation and western blotting}

Immunoprecipitation and western blotting were performed as previously described [23].

\section{Patient samples and IHC}

Paraffin-embedded SCC tissue blocks were collected from 81 patients during their initial treatment at Zhejiang Cancer Hospital. Written informed consent was obtained from all patients. IHC was performed as previously described [22]. IHC staining was calculated as an $H$-score, as follows: $H$-score $=\Sigma$ pi $\times \mathrm{i}$, where pi represents the percentage of positive cells $(0-100 \%)$ and $i$ represents the staining intensity ( 0 : negative; 1 : weak; 2 : medium; and 3 : strong). IHC staining was scored by two independent observers.

\section{ED-L2-Cre/ARID1A loxp/loxp $^{\text {mice and 4-nitroquinoline }}$ 1-oxide (4NQ0) treatment}

All animal studies were approved by the Animal Care and Use Committee of the Chinese Academy of Medical Sciences Cancer Hospital. ED-L2-Cre mice were generated as previously described [24]. Aridla $a^{\text {tml.IZhwa }}$ mice were purchased from The Jackson Laboratory (stock \#027717, ME, USA). The mutant mice were homozygous for floxed Aridla and hemizygous for the Cre transgene, and littermate Aridla $a^{\operatorname{loxp} /}$ loxp mice lacking the Cre transgene served as controls. All mice used for this study were on a C57BL/6J Gpt genetic background. Mice were administered 4NQO (Sigma-Aldrich,
MO, USA) $(100 \mu \mathrm{g} / \mathrm{ml})$ in drinking water from the age of 6 weeks. After $4 \mathrm{NQO}$ treatment for 16 weeks, mice were supplied drinking water without $4 \mathrm{NQO}$ until sacrifice.

\section{Xenograft transplantation experiments}

All animal protocols were approved by the Animal Care and Use Committee of the Chinese Academy of Medical Sciences Cancer Hospital. For the tumor formation assay, $1 \times$ $10^{6}$ Het-1A or NE-3 cells premixed with Matrigel Matrix (Corning, NY, USA) at a ratio of $1: 1$ were injected subcutaneously into 6-week-old male NCG mice (GemPharmatech Co., Ltd, Nanjing, China). For subcutaneous xenografting, $1-4 \times 10^{6}$ ESCC cells were injected subcutaneously into 6-week-old male BALB/c nude mice. DDP $(6 \mathrm{mg} / \mathrm{kg})$ was administered three times a week until sacrifice. All mice were randomly allocated to different groups before further treatment. Tumor size was calculated as $0.52 \times$ length $\times$ width $^{2}$.

\section{Virus production and cell infection}

Lentivirus was produced using $293 \mathrm{~T}$ cells with the secondgeneration packaging system psPAX2 (\#12260, Addgene, MA, USA) and the pMD2.G plasmid (\#12259, Addgene). Transfections were performed using Hieff Trans Liposomal Transfection Reagent (40802, Yeasen, Shanghai, China) according to the manufacturer's protocol. Cells were infected with lentivirus supplemented with $8 \mu \mathrm{g} / \mathrm{ml}$ polybrene (Sigma-Aldrich) twice within $48 \mathrm{~h}$ and then selected with $1 \mu \mathrm{g} / \mathrm{ml}$ puromycin (Sangon Biotech, Shanghai, China) or $200 \mu \mathrm{g} / \mathrm{ml} \mathrm{G} 418$ (MedChemExpress) for 10 days. The short hairpin RNA (shRNA) sequences targeting ARID1A were as follows: sh1, 5'-GCCTGATCTATCTGGTTCA AT-3', and sh2, 5'-GCATCCTTCCATGAACCAATC-3'. The shRNA sequences targeting c-Myc were as follows: sh1, 5'-GGAAACGACGAGAACAGTTGA-3', and sh2, 5'-CCTGAGACAGATCAGCAACAA-3'. The following nontargeting shRNA sequence was used as the negative control: 5'-AGTCTTAATCGCGTATAAGGC-3'.

\section{CCK-8 cell proliferation assays}

CCK-8 reagent (Dojindo, Tokyo, Japan) was added to the cell culture medium at a ratio of 1:10 preuse, and the absorbance was measured at $450 \mathrm{~nm}$ to assess cell viability after incubation at $37^{\circ} \mathrm{C}$ for $1 \mathrm{~h}$.

\section{Plate clonality and soft agar colony formation assays}

For the plate clonality assays, cells were plated into sixwell plates at a density of 1500 cells per well and 
A
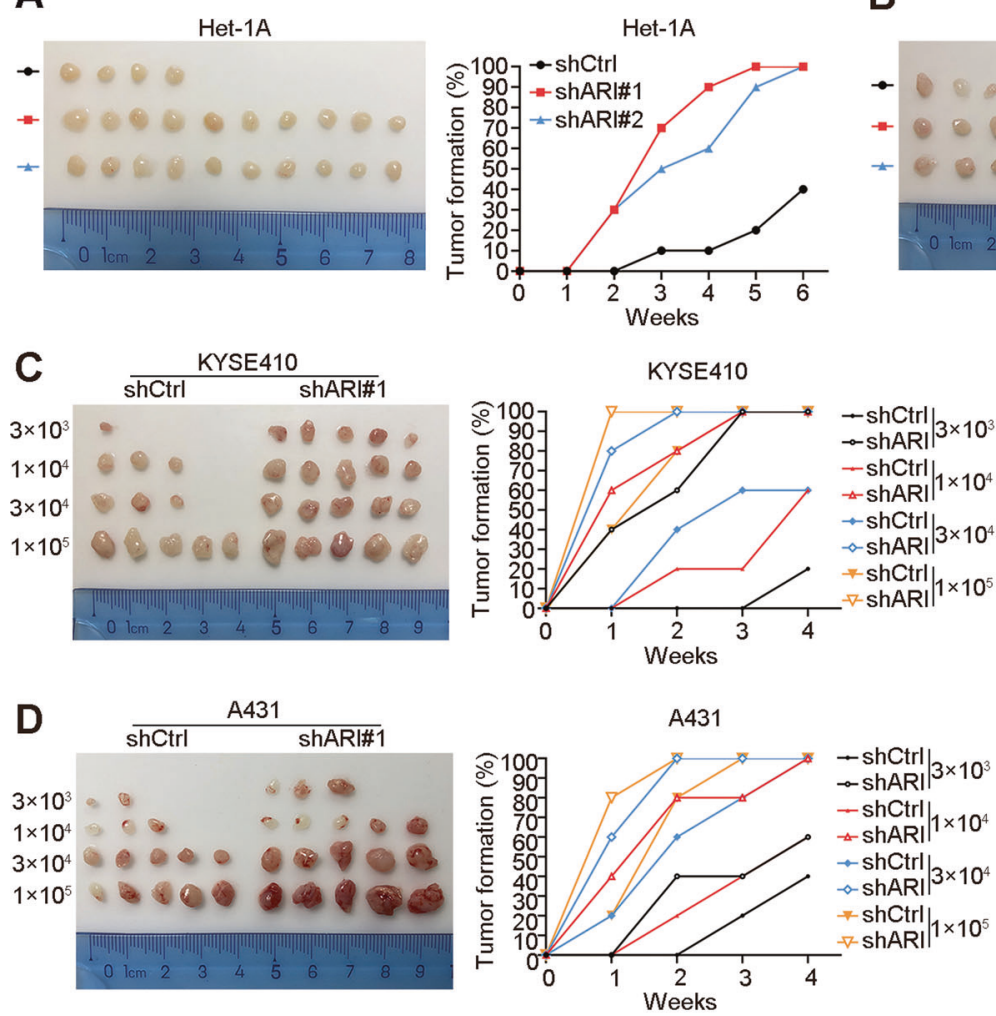

E

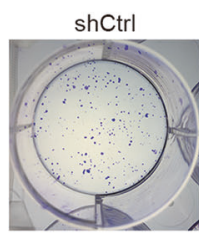

ShARI\#1

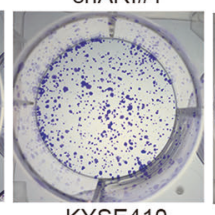

KYSE410
ShARI\#2

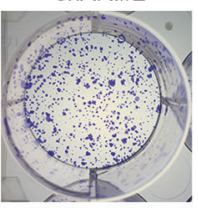

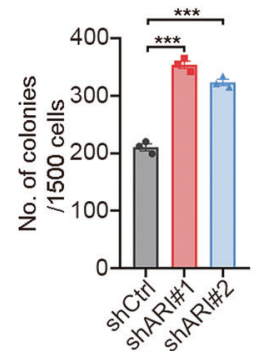

B
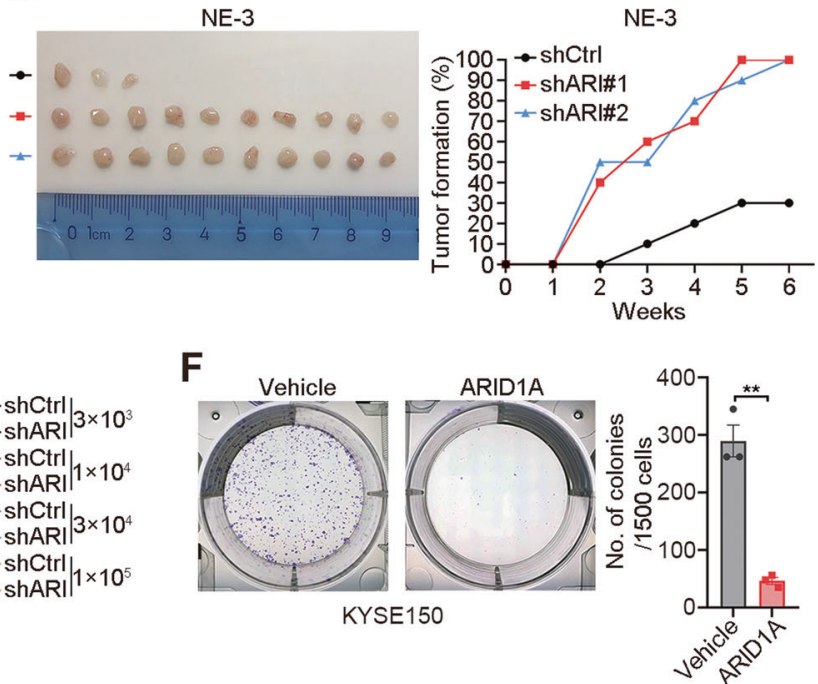

G
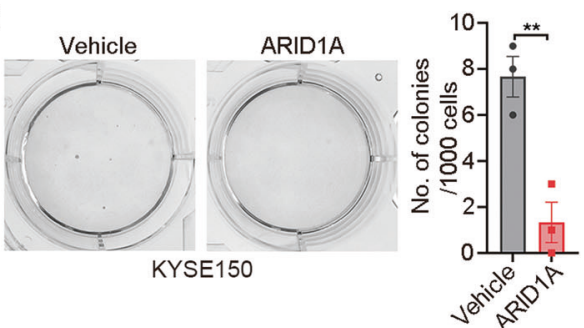

H
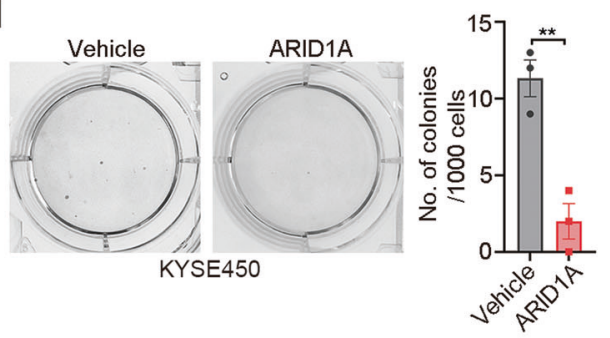

Fig. 2 ARID1A depletion promotes tumorigenesis in human squamous cell carcinoma cells. a, b Tumor formation rates of Het-1A and NE-3 cells expressing control (shCtrl) or ARID1A shRNAs (shARI\#1, shARI\#2) ( $n=10$ per group). $\mathbf{c}, \mathbf{d}$ Tumor formation rates of KYSE410 and A431 cells expressing control (shCtrl) or ARID1A shRNA (shARI\#1) ( $n=5$ per group). e Representative images and numbers of colonies formed by KYSE410 cells expressing control
(shCtrl) or ARID1A shRNAs (shARI\#1, shARI\#2). f Representative images and numbers of plate colonies formed by KYSE150 cells expressing the empty vector (Vehicle) or ARID1A. Representative images and numbers of soft agar colonies formed by KYSE150 (g) or KYSE450 (h) cells expressing the empty vector (Vehicle) or ARID1A. Data are shown as the mean \pm s.e.m.; $n=3$ independent experiments, two-tailed $t$ tests; $* * P<0.01, * * * P<0.001$. incubated for 2 weeks. The colonies were then stained with $0.4 \%$ crystal violet dissolved in methyl alcohol for $30 \mathrm{~min}$. For the soft agar colony formation assays, the wells were precoated with $0.5 \%$ noble agar (SigmaAldrich), and 1000 cells were mixed with $0.35 \%$ noble agar and then plated into the wells. Colonies were counted and imaged after 3 weeks.

\section{Spheroid formation assays}

A total of 1000 cells were cultured in suspension in a sixwell plate with an ultralow attachment surface (Corning).
Serum-free DMEM/F12 medium supplemented with insulin (4 $\mu \mathrm{g} / \mathrm{ml}$, Sigma-Aldrich), B27 (1:50, Invitrogen), human recombinant EGF ( $20 \mathrm{ng} / \mathrm{ml}$, Sigma-Aldrich), and human recombinant basic FGF (10 ng/ml, Invitrogen) was used to induce spheroid formation. Cells were replenished with $200 \mu \mathrm{l}$ of supplemented medium every other day, and the number of spheroids formed was counted after 2 weeks.

\section{Flow cytometry}

Cell apoptosis was detected by staining with annexin V-FITC/propidium iodide (PI) using an apoptosis detection 
Fig. 3 ARID1A depletion promotes cancer stemness. a Immunoblotting analysis of the indicated proteins in KYSE410 and A431 cells expressing control (shCtrl) or ARID1A shRNAs (shARI\#1, shARI\#2). b Immunoblotting analysis of the indicated proteins in KYSE150 and KYSE450 cells expressing the empty vector (Vehicle) or ARID1A. c, $\mathbf{d}$ Representative images and numbers of spheroids formed by KYSE410 cells expressing control (shCtrl) or ARID1A shRNAs (shARI\#1, shARI\#2).

e, f Representative images and numbers of spheroids formed by A431 cells expressing control (shCtrl) or ARID1A shRNAs (shARI\#1, shARI\#2). $\mathbf{g}, \mathbf{h}$ Representative images and numbers of spheroids formed by KYSE150 cells expressing the empty vector (Vehicle) or ARID1A. i, j Representative images and numbers of spheroids formed by KYSE450 cells expressing the empty vector (Vehicle) or ARID1A. Scale bars, $100 \mu \mathrm{m}$. Data are shown as the mean \pm s.e.m.; $n=$ 3 independent experiments, two-tailed $t$ tests; $* P<0.05$, $* * P<0.01$.
A

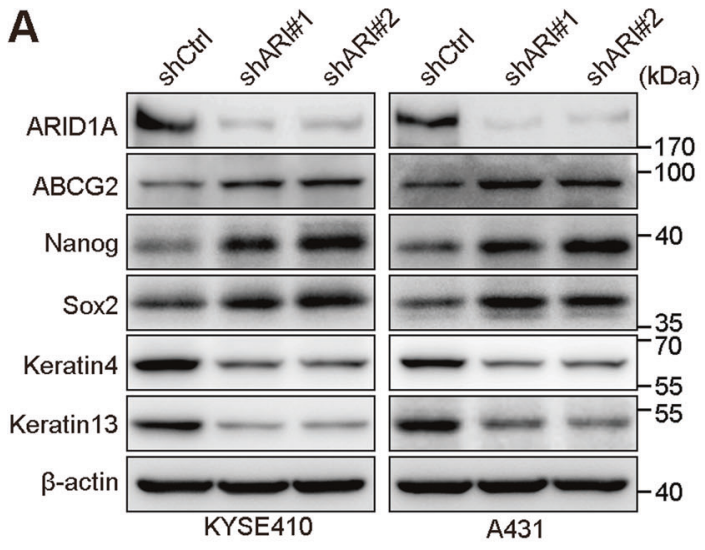

C

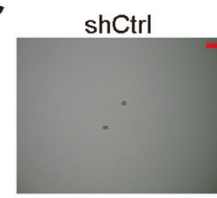

E

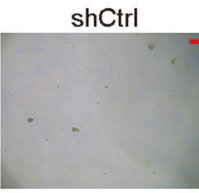

G

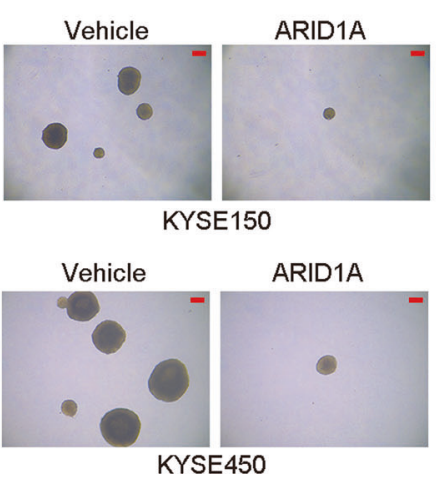

B

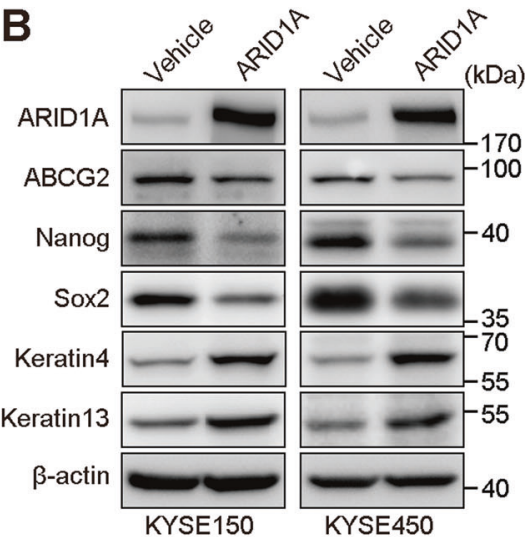

D

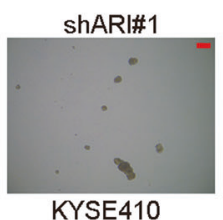

shARI\#2

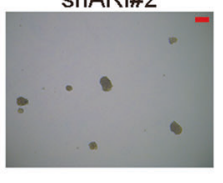

ShARI\#1
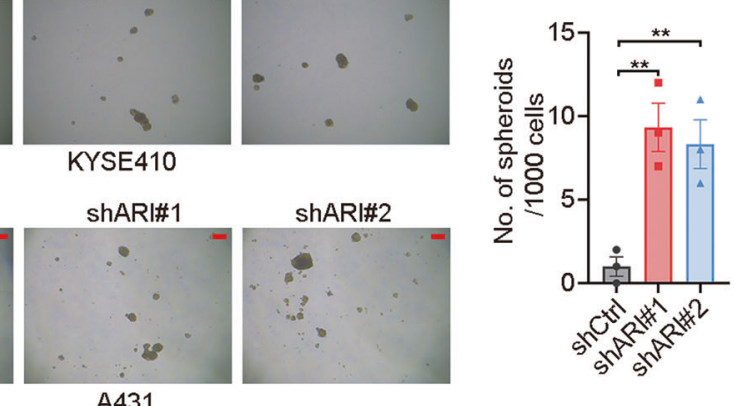

$\mathbf{F}$

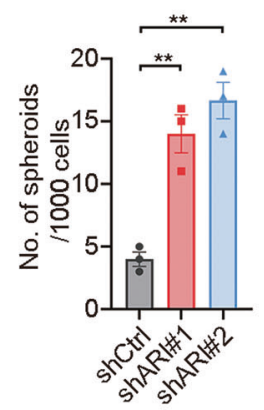

kit (Dojindo, Tokyo, Japan) according to the manufacturer's protocol.

\section{RNA sequencing and gene set enrichment analysis (GSEA)}

Three pairs of samples were generated from three biologically independent experiments. RNA-seq libraries were prepared using the NEBNext ${ }^{\circ}$ Ultra RNA Library Prep Kit for Illumina (New England Biolabs, MA, USA) and validated using the 2100 Bioanalyzer (Agilent Technologies). Libraries were sequenced on a NovaSeq 6000 sequencer (Illumina, CA, USA). Clean reads were aligned to the human genome (hg37) using HISAT2 (version 2.0.5) after removing low-quality reads. The differential expression of
ARID1A between ARID1A-depleted and control cells was computed using the fragments per kilobase of transcript per million mapped reads calculated by featureCounts (version 1.5.0-p1). Pathway analyses of differentially expressed genes were performed using GSEA as previously described [25]. The enrichment of differentially expressed genes in ARID1A-depleted and control A431 cells was carried out using MSigDB hallmark gene sets. The RNA-seq data can be accessed in the Gene Expression Omnibus database under accession number GSE131132.

\section{Statistics}

All mice or samples were included in our analysis. Pilot experiments and previous published results were used to 
A EPITHELIAL_MESENCHYMAL
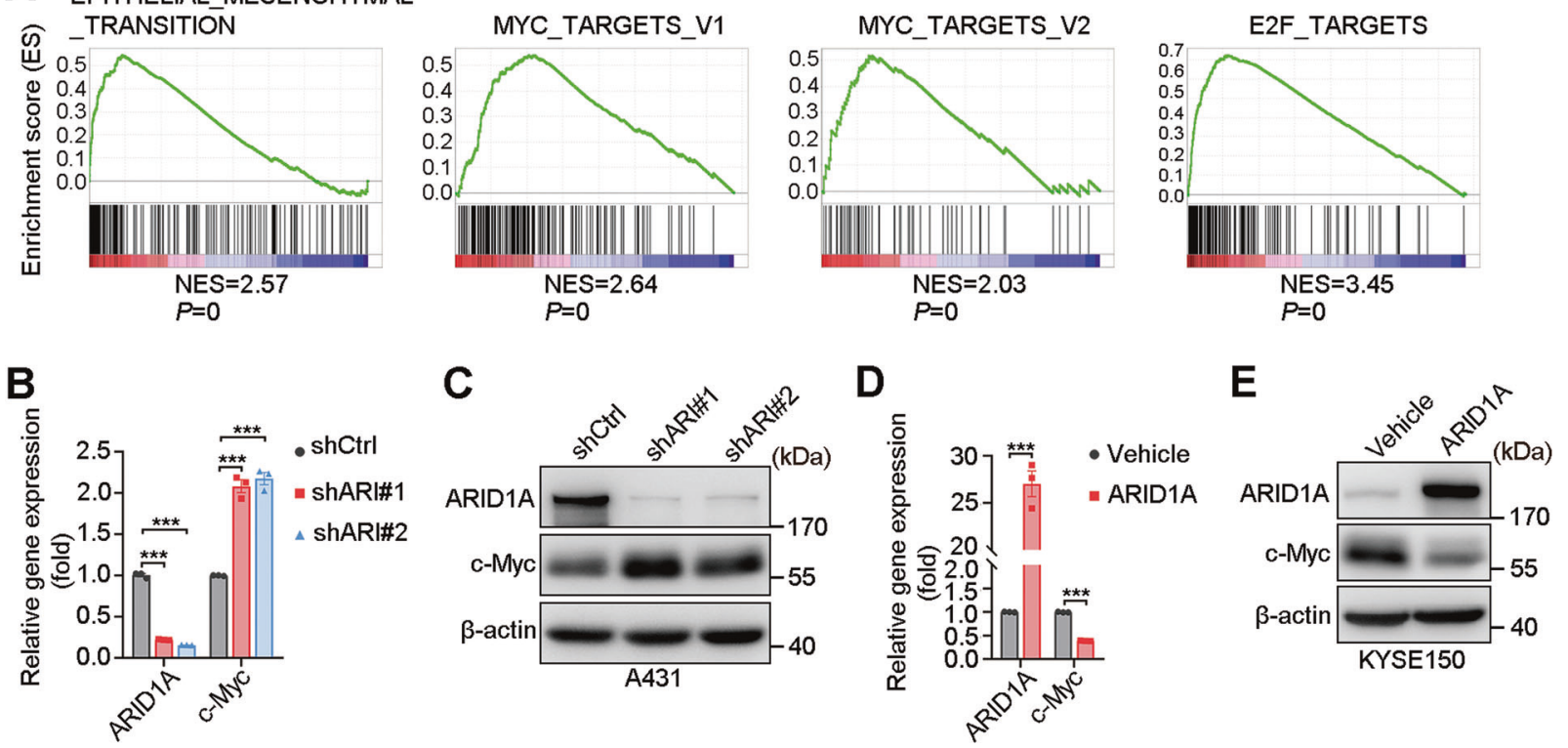

$\mathbf{F}$

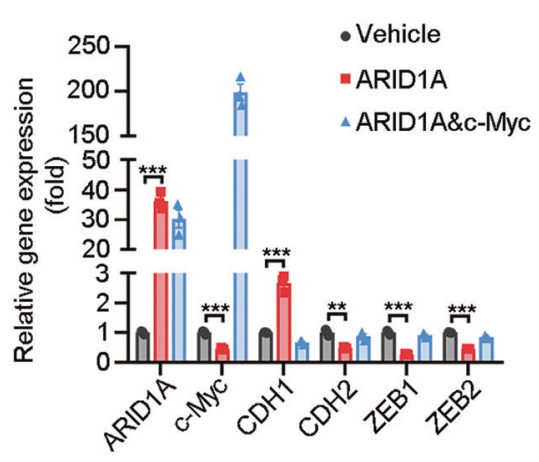

H

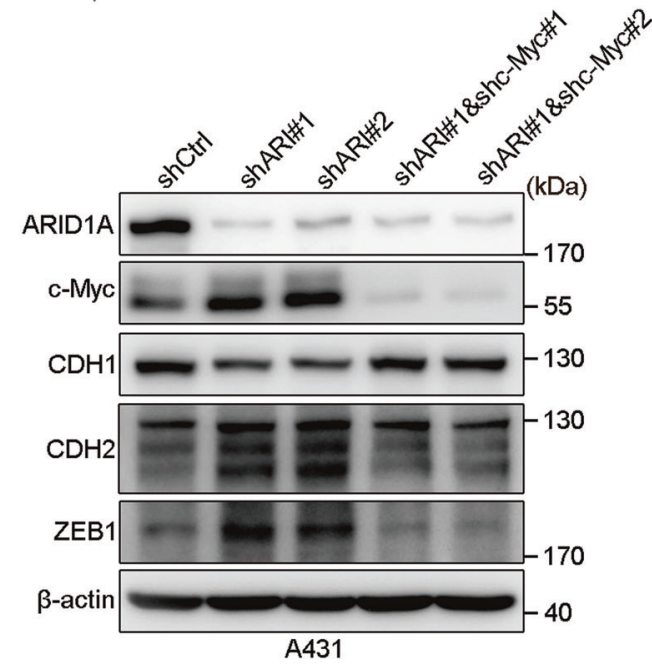

estimate the proper sample size. The exact $n$ numbers used in the study are indicated in the respective figure legends. The investigators were blinded to group allocation during data collection and analysis. For all statistical analyses, differences
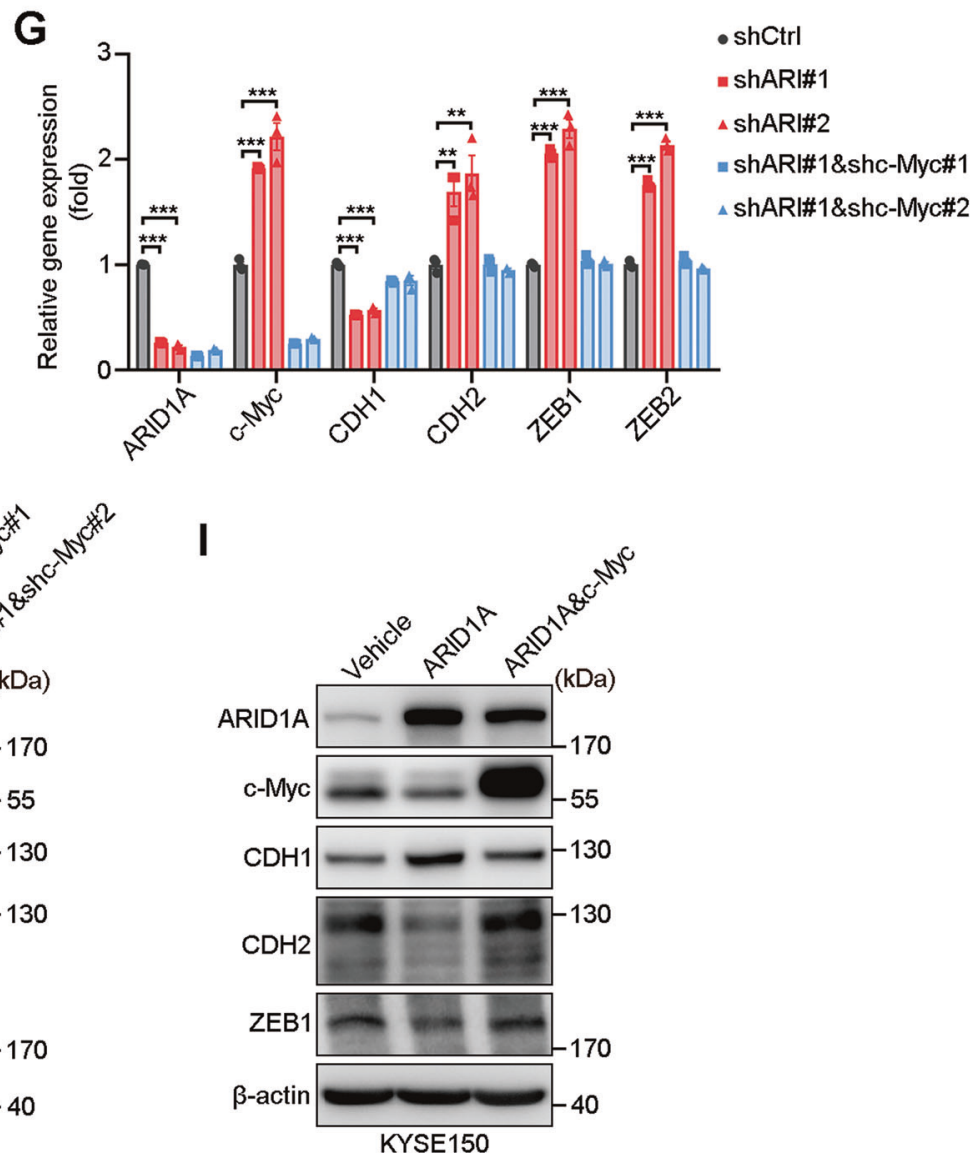

with $P \leq 0.05$ were considered statistically significant, and data from at least three biologically independent experiments with similar results are shown. Data analysis was performed using GraphPad Prism software (version 8.0). 
Fig. 4 ARID1A depletion promotes EMT by activating c-Myc. a GSEA of the top enriched pathways in ARID1A-depleted A431 cells compared with control cells based on the RNA sequencing data (cells from $n=3$ independently grown cultures). b, c qRT-PCR and immunoblotting analysis of the expression of the indicated genes in A431 cells expressing control (shCtrl) or ARID1A shRNAs (shARI\#1, shARI\#2). d, e qRT-PCR and immunoblotting analysis of the expression of the indicated genes in KYSE150 cells expressing the empty vector (Vehicle) or ARID1A. f, $\mathbf{g}$ qRT-PCR analysis of the expression of the indicated genes in KYSE150 cells expressing the empty vector (Vehicle) or ARID1A or ARID1A with ectopic c-Myc, and A431 cells expressing control (shCtrl), ARID1A shRNAs (shARI\#1, shARI\#2), or ARID1A shRNAs together with c-Myc shRNAs (shARI\#1\&shc-Myc\#1, shARI\#1\&shc-Myc\#2). h, i Immunoblotting analysis of the expression of the indicated genes in A431 cells expressing control (shCtrl) or ARID1A shRNAs (shARI\#1, shARI\#2) or ARID1A shRNAs together with c-Myc shRNAs (shARI\#1\&shc-Myc\#1, shARI\#1\&shc-Myc\#2), and KYSE150 cells expressing the empty vector (Vehicle) or ARID1A or ARID1A with ectopic c-Myc. Data are shown as the mean \pm s.e.m.; $n=3$ independent experiments, two-tailed $t$ tests; $* * P<0.01, * * * P<0.001$.

\section{Results}

\section{Arid1a knockout promotes SCC initiation in mice}

To explore the role of ARID1A in SCC initiation, we first crossed ED-L2-Cre mice [24] with Aridla ${ }^{\text {tml.IZhwa mice }}$ [26]. The specific expression of Cre recombinase enabled the deletion of ARID1A expression within the epithelia of the tongue, esophagus, and forestomach in the generated ED-L2-Cre/Aridla $a^{\text {loxp/loxp }}$ mice. Then, we utilized an oralesophageal carcinogen, 4NQO [27], to mimic environmental factors that cause the development of SCC (Fig. 1a). Until 28 weeks of age, both the homozygous and heterozygous knockout mice exhibited significantly poorer prognoses than the wild-type mice (Fig. 1b), illustrating that the decrease in ARID1A expression, instead of a complete inactivating mutation, is sufficient to promote SCC initiation and contributes to a poor prognosis. To further study the pro-tumorigenesis function of ARID1A loss, mice were collected at 24, 28, and 32 weeks old to evaluate the tumor initiation status (Fig. 1a). The number of tongue tumors increased obviously from 24-week-old mice to 28 -week-old mice, while there was no significant increase after 28 weeks of age, indicating that 28 weeks is likely the most effective time point for 4NQO-induced SCCs (Fig. 1c, d). Consistent with the survival outcomes, the number of tumors on the tongues of both homozygous and heterozygous knockout mice was significantly higher than those of wild-type mice at all three time points examined (Fig. 1c, d). Moreover, at 32 weeks, 4NQO-induced wild-type mice had a tumor incidence of $42.9 \%$, while the incidence of esophageal tumors in both homozygous and heterozygous knockout mice reached $100 \%$ (Fig. 1e, f). No spontaneous tumorigenesis was found in Aridla knockout mice till 32 weeks, indicating the loss of ARID1A alone might be insufficient for the development of SCC.

The ablation of ARID1A expression in knockout mice was confirmed by IHC experiments (Fig. 1g, h). Moreover, there was stronger Ki-67 staining in the esophageal epitheliums of knockout mice than in wild-type mice (Fig. 1g, h), supporting our former results that ARID1A loss promotes the malignant transformation of squamous epithelial cells. Interestingly, we observed that though ARID1A expression was relatively high in the normal esophageal epithelium, it was frequently absent in the basal layer of the esophagus (Fig. 1g). Cells in the basal layer have stem cell features and are responsible for epithelial differentiation and development [28]. Thus, we hypothesized that ARID1A depletion might promote SCC initiation by increasing cancer stemness. To confirm our speculation, IHC staining was performed to detect the expression of Sox2, a wellrecognized stemness marker of SCCs [29]. The epithelial cells in wild-type mice showed negative Sox 2 expression, while the epithelial cells in ARID1A knockout mice showed elevated Sox2 expression (Fig. 1g, h).

\section{ARID1A depletion promotes SCC initiation by increasing cancer stemness}

To study the role of ARID1A in SCC initiation in human cells, we knocked down ARID1A expression using two independent shRNAs in two immortalized human esophageal epithelial cell lines, Het-1A and NE-3 (Supplementary Fig. 1a). Then, we xenografted control and ARID1Adepleted Het-1A and NE-3 cells into NCG mice. Both immortalized human esophageal epithelial cells gained potent tumor formation ability after ARID1A depletion (Fig. 2a, b). To further validate the role of ARID1A in the tumorigenic potential of SCC cells, a limiting dilution analysis was performed using KYSE410 cells expressing control or ARID1A shRNAs. ARID1A-depleted KYSE410 cells reached a tumor formation rate of $100 \%(5 / 5)$, even at a transplantation number of $3 \times 10^{3}$ cells per mouse, while control KYSE410 cells reached a tumor formation rate of only $20 \%(1 / 5)$ at the same dilution rate (Fig. 2c and Supplementary Fig. 1b). Using another SCC cell line, A431, we also validated significantly increased tumorigenic potential after ARID1A depletion (Fig. 2d and Supplementary Fig. 1b). Consistent with these results, ARID1A-depleted KYSE410 cells displayed increased colony formation ability compared with control cells On the other hand, the overexpression of ARID1A in KYSE150 and KYSE450 cells nearly abolished their colony formation abilities in both colony formation and soft agar assays (Fig. $2 \mathrm{f}-\mathrm{h}$ and Supplementary Fig. 1c).

To validate whether ARID1A depletion promotes SCC initiation by increasing cancer stemness, we first performed 
A

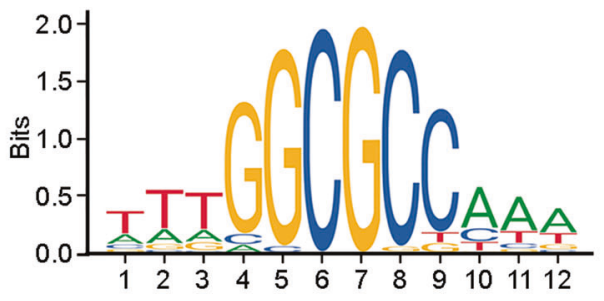

B

\begin{tabular}{lll}
\hline Name & Predicted sequence & Score \\
\hline E2F1 & TTTGCTGCCAAA & 8.02522 \\
E2F1 & TGTGGCGCCGGT & 7.64724 \\
E2F1 & ACCGGCGCCACA & 7.47893 \\
E2F1 & CGAGGCGCCCTG & 7.17753 \\
E2F1 & TTTGGCAGCAAA & 6.92855 \\
E2F1 & GTGGGCGCGCAG & 6.9188 \\
E2F1 & ATAAGCGCCCCT & 6.80046 \\
\hline
\end{tabular}

C

Chromosome 8

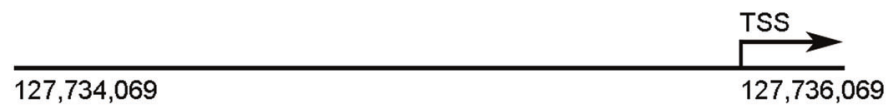

E2F1 binding sites

(ENCODE) $\quad(127,733,934-127,734,433)$

CHIP-qPCR primer

$$
\text { c-Myc-P1 }
$$

D

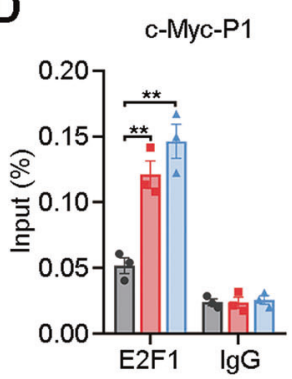

$\mathbf{F}$

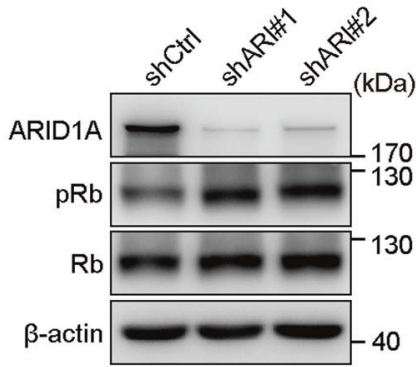

G

$(127,735,203-127,735,702)$
c-Myc-P2

E
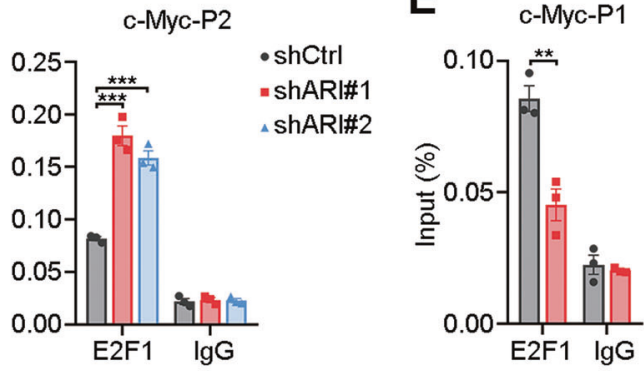

H Myc-Rb - +

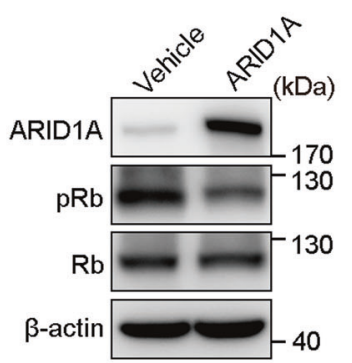

V5-ARID1A
$(127,736,016-127,736,328)$
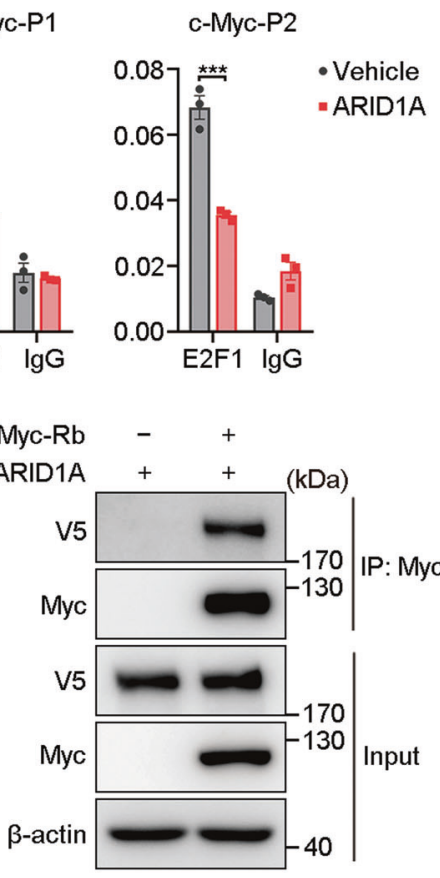

I

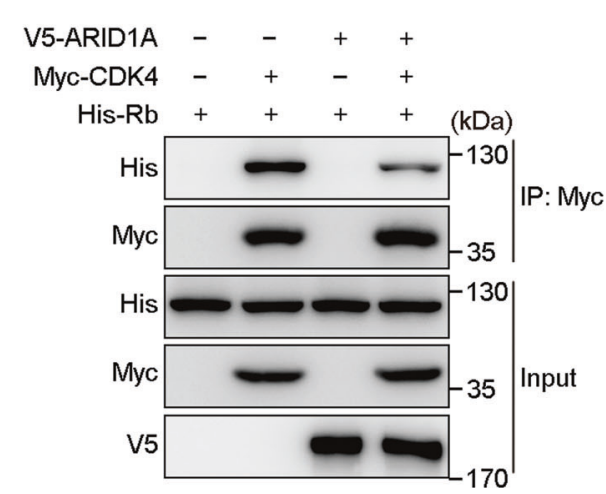

J

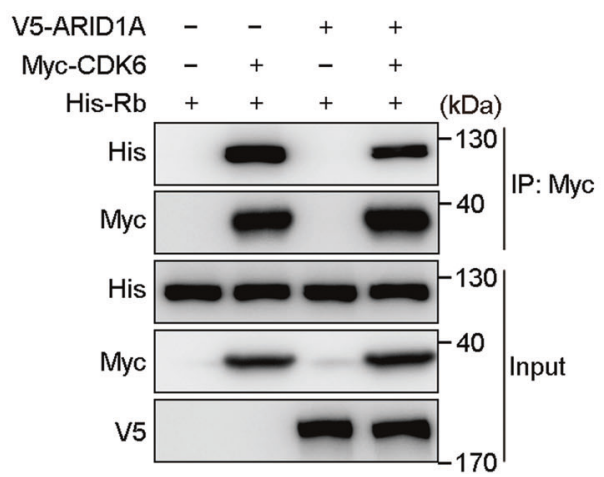

immunoblotting to assess the expression of stemness and differentiation markers in SCC cell lines. ARID1A depletion increased the expression of stemness markers such as
ABCG2, Nanog, and Sox 2 and decreased the expression of differentiation markers such as Keratin 4 and Keratin 13, and the ectopic expression of ARID1A resulted in the 
Fig. 5 ARID1A prevents Rb phosphorylation to suppress c-Myc transcription. a Prediction of the sequence logo of E2F1 by the JASPAR database. $\mathbf{b}$ The binding sites of E2F1 on the c-Myc promoter region were predicted by JASPAR. c Schematics showing three regions of $\mathrm{E} 2 \mathrm{~F} 1$ enriched at the $\mathrm{c}-\mathrm{Myc}$ promoter region by the ENCODE database. d ChIP analysis of A431 cells expressing control (shCtrl) or ARID1A shRNAs (shARI\#1, shARI\#2) using antibodies against E2F1 for the indicated gene promoters. e ChIP analysis of KYSE150 cells expressing the empty vector (Vehicle) or ARID1A using antibodies against E2F1 for the indicated gene promoters. f, $\mathbf{g}$ Immunoblotting analysis of the indicated proteins in A431 cells expressing control (shCtrl) or ARID1A shRNAs (shARI\#1, shARI\#2) and KYSE150 cells expressing the empty vector (Vehicle) or ARID1A. h-j Immunoblotting analysis of the indicated proteins in coimmunoprecipitation assays performed using anti-Myc magnetic beads in 293T cells expressing the indicated plasmids. Data are shown as the mean \pm s.e.m.; $n=3$ independent experiments, two-tailed $t$ tests; $* * P<0.01, * * * P<0.001$.

opposite effects (Fig. 3a, b). To further confirm the effect of ARID1A on cancer stemness in SCC, spheroid formation assays were performed. ARID1A depletion in KYSE410 and A431 cells enhanced spheroid formation ability (Fig. 3c-f). Consistent with these findings, the ectopic expression of ARID1A suppressed the spheroid formation ability of both KYSE150 and KYSE450 cells (Fig. 3g-j). These evidences illustrate that ARID1A depletion promotes SCC initiation by increasing cancer stemness.

\section{ARID1A depletion activates c-Myc to promote epithelial-to-mesenchymal transition (EMT)}

To study the specific mechanism by which ARID1A loss promotes SCC tumorigenesis, RNA sequencing was performed using A431 cells expressing control or ARID1A shRNAs. GSEA was performed to enrich functionally related pathways, and EMT and c-Myc targets were among the top enriched pathways (Fig. 4a). Interestingly, c-Myc is a well-known oncogene that drives cancer stemness and tumorigenesis by inducing EMT [30, 31]. We speculated that ARID1A depletion might promote the cancer stemness and tumor initiation of SCC cells by activating c-Myc expression. To validate our speculation, we first knocked down ARID1A expression in A431 cells and performed immunoblotting and qRT-PCR to examine the expression level of c-Myc. ARID1A depletion increased c-Myc expression at both the mRNA and protein levels (Fig. 4b, c), supporting the oncogenic functions following ARID1A depletion. Consistent with these results, the overexpression of ARID1A in KYSE150 cells inhibited c-Myc mRNA and protein expression (Fig. 4d, e).

We next sought to validate whether ARID1A depletion promotes EMT via c-Myc activation. We first overexpressed ARID1A with or without ectopically expressed cMyc protein in KYSE150 cells. qRT-PCR analysis showed increased $\mathrm{CDH} 1$ expression as well as decreased $\mathrm{CDH} 2$,
ZEB1, and ZEB2 expression after ARID1A overexpression, which was rescued upon c-Myc overexpression (Fig. 4f). Consistent with these findings, ARID1A knockdown by two independent shRNAs in A431 cells increased the expression of the mesenchymal markers CDH2, ZEB1, and ZEB2 and decreased CDH1 expression by activating c-Myc expression, which could be reversed by knocking down c-Myc (Fig. 4g). Immunoblotting assays further confirmed that ARID1A depletion increased $\mathrm{CDH} 2$ and ZEB1 expression but decreased $\mathrm{CDH} 1$ expression by activating c-Myc Moreover, the ectopic expression of c-Myc abolished mesenchymal-to-epithelial transition (MET) caused by ARID1A overexpression (Fig. 4i). These results validate that c-Myc is the downstream target of ARID1A that mediates EMT by ARID1A loss.

\section{ARID1A suppresses c-Myc transcription by preventing Rb phosphorylation}

To specify the regulatory mechanism of ARID1A in c-Myc transcription, we first analyzed the RNA sequencing data to investigate whether some transcriptional events were involved in the differentially expressed genes following ARID1A depletion. Surprisingly, we noticed that E2F targets were one of the most enriched pathways in our RNA sequencing data (Fig. 4a). Next, we predicted the sequence logo of E2F1 using the JASPAR database [32] (Fig. 5a), and further analysis showed that E2F1 could bind to several sequences in the c-Myc promoter region ( $2 \mathrm{~kb}$ upstream of the transcriptional start site) (Fig. 5b). Further analysis using the ENCODE database in the UCSC Genome Browser revealed three regions that showed E2F1 enrichment at the c-Myc promoter region (Fig. 5c). These results suggest that ARID1A might regulate c-Myc expression by affecting the transcriptional activity of E2F1. We next validated whether E2F1 indeed acts as a transcription factor of c-Myc in SCC cells. We first designed two pairs of ChIPqPCR primers based on the regions provided by the ENCODE database (Fig. 5c), and ChIP assays were performed using an anti-E2F1 antibody in A431 cells expressing control or ARID1A shRNAs. The results showed that the c-Myc promoter region was significantly immunoprecipitated compared with the IgG group (Fig. 5d). Moreover, the association between $\mathrm{E} 2 \mathrm{~F} 1$ and the $\mathrm{c}-\mathrm{Myc}$ promoter region increased significantly in ARID1A-depleted cells compared with control cells (Fig. 5d). On the other hand, ARID1A overexpression in KYSE150 cells decreased the association between E2F1 and the c-Myc promoter region (Fig. 5e).

The $\mathrm{CDK} / \mathrm{Rb}$ pathway plays an essential role in regulating the transcriptional activity of E2F1, in which phosphorylated $\mathrm{Rb}(\mathrm{pRb})$ loses its suppression on E2F1 and leads to the activation of E2F1 targets [33, 34]. To explore 
A

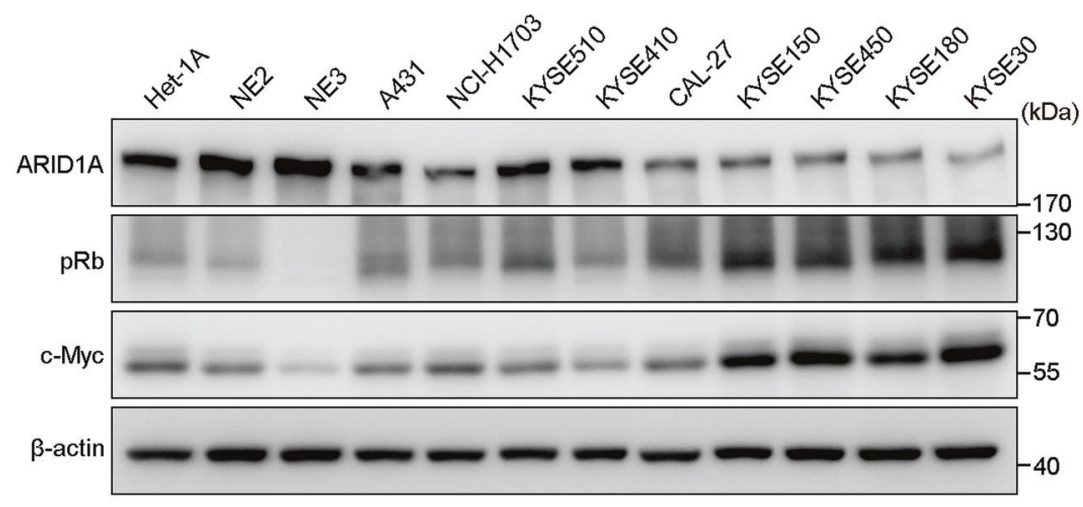

B
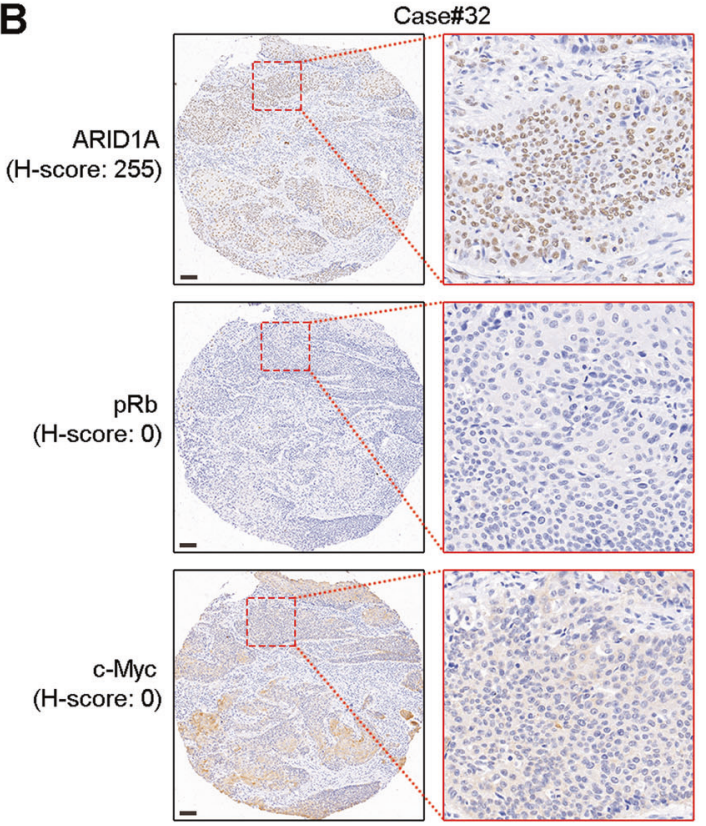

D

\begin{tabular}{lccc}
\hline & \multicolumn{3}{c}{ ARID1A } \\
\cline { 2 - 3 } $\mathrm{pRb}$ & Low & High & Total \\
\hline Low & 15 & 35 & 50 \\
High & 19 & 12 & 31 \\
Total & 34 & 47 & 81 \\
\hline \multicolumn{4}{c}{$P=0.0055$}
\end{tabular}

G

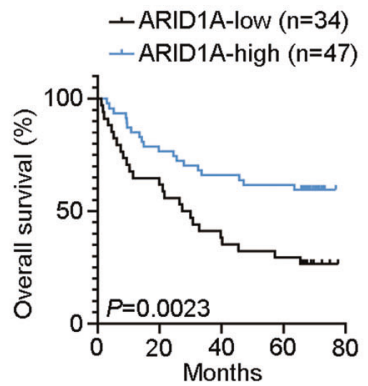

C Case\#42
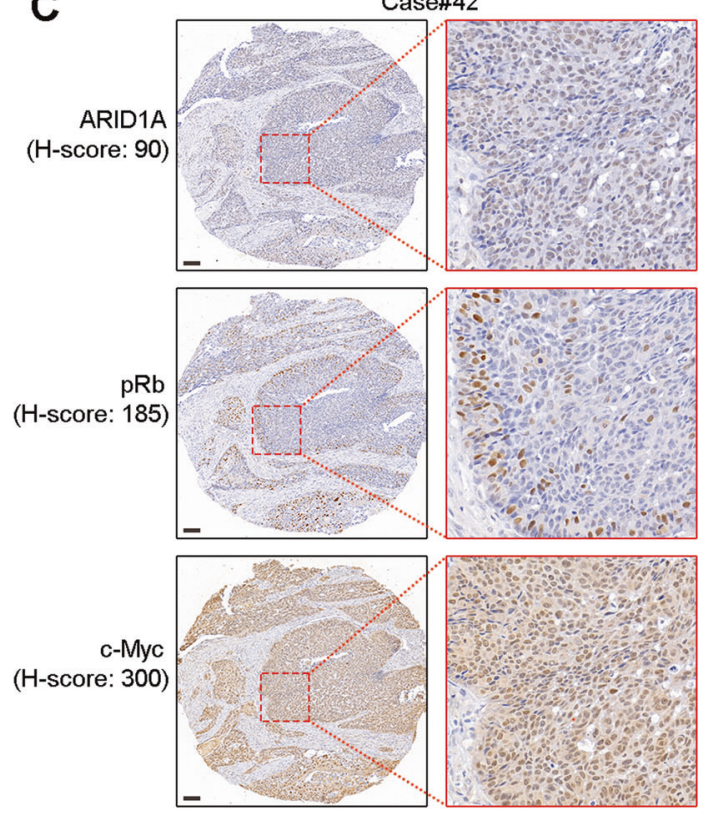

$\mathbf{F}$
$\mathbf{H}$

\begin{tabular}{lccc}
\hline & \multicolumn{2}{c}{ ARID1A } & \\
\cline { 2 - 3 } c-Myc & Low & High & Total \\
\hline Low & 11 & 35 & 46 \\
High & 23 & 12 & 35 \\
Total & 34 & 47 & 81 \\
\hline \multicolumn{4}{c}{$P=0.0002$}
\end{tabular}

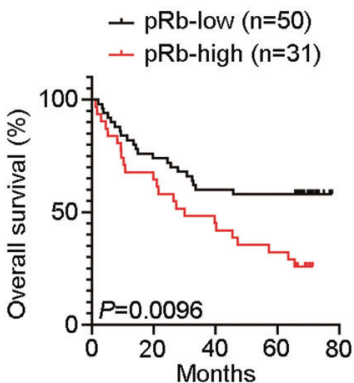

\begin{tabular}{lccc}
\hline & \multicolumn{2}{c}{$\mathrm{pRb}$} & \\
\cline { 2 - 3 } c-Myc & Low & High & Total \\
\hline Low & 35 & 11 & 46 \\
High & 15 & 20 & 35 \\
Total & 50 & 31 & 81 \\
\hline \multicolumn{4}{c}{$P=0.0023$}
\end{tabular}

I

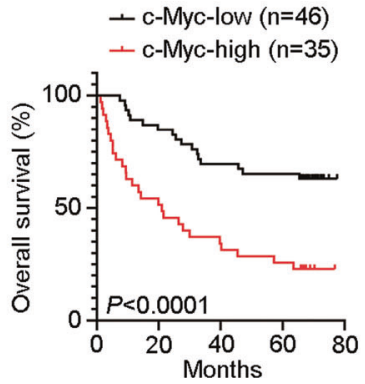

Fig. 6 ARID1A expression is negatively correlated with $\mathrm{pRb} / \mathrm{c}$ Myc expression. a Immunoblotting analysis of ARID1A, pRb, and cMyc expression in the indicated cell lines. $\mathbf{b}$, $\mathbf{c}$ Representative images of IHC staining for ARID1A, pRb, and c-Myc expression in SCC samples. d-f $H$-scores and correlation analysis of IHC staining for
ARID1A, pRb, and c-Myc expression in SCC samples. Chi-square tests. g-i Kaplan-Meier analysis of SCC patients with low- or highARID1A expression, low- or high-pRb expression and low- or high-cMyc expression. Scale bars, $100 \mu \mathrm{m}$. 
A

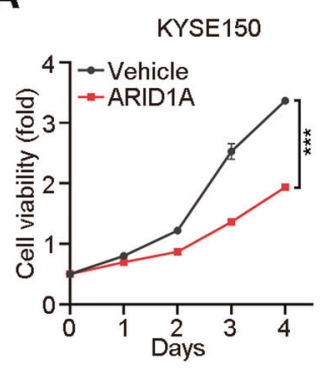

B

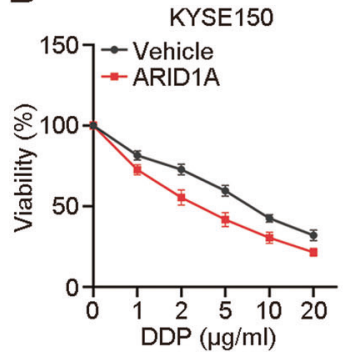

$\mathbf{E}$

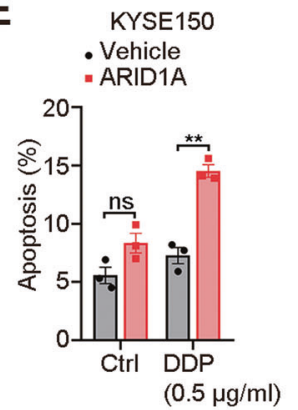

G

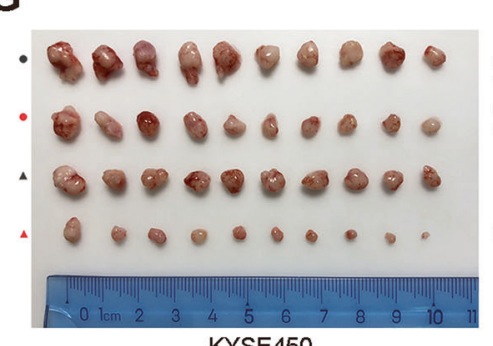

KYSE450

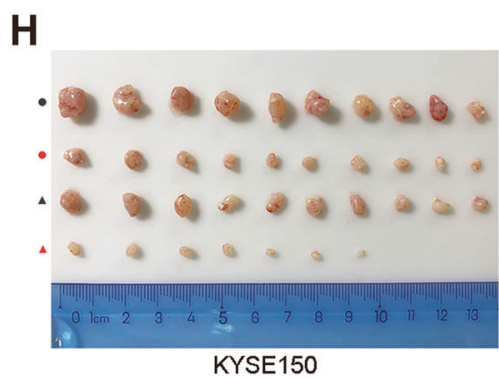

KYSE150

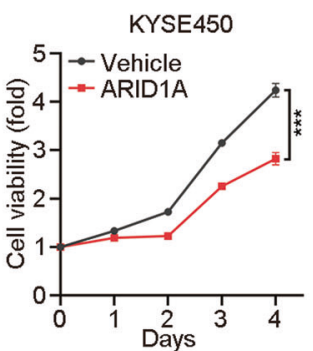

C
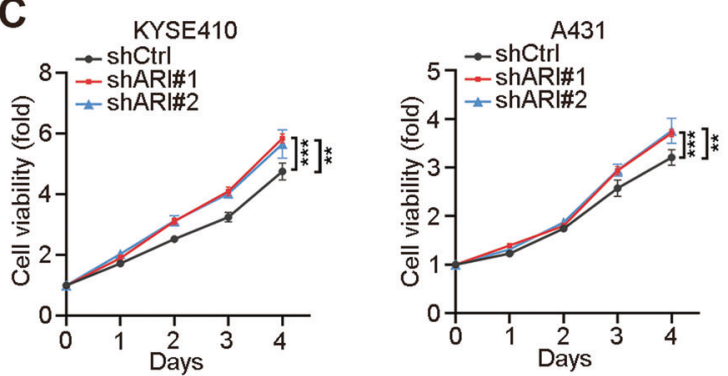

D
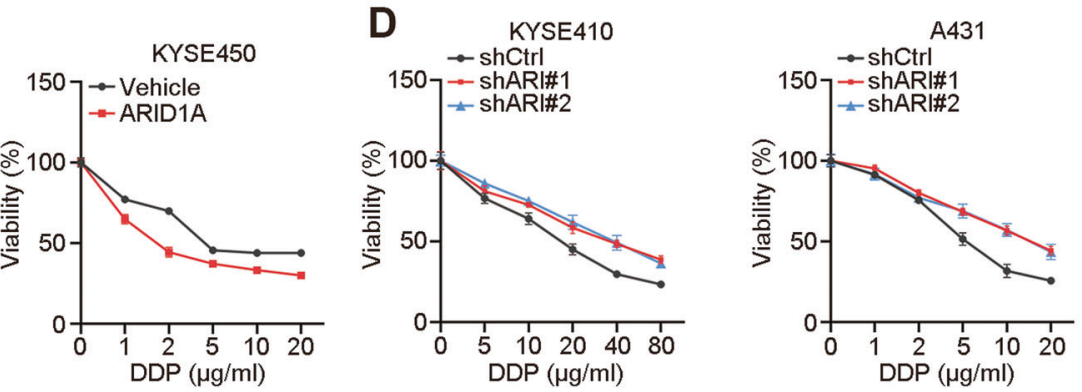

$\mathbf{F}$
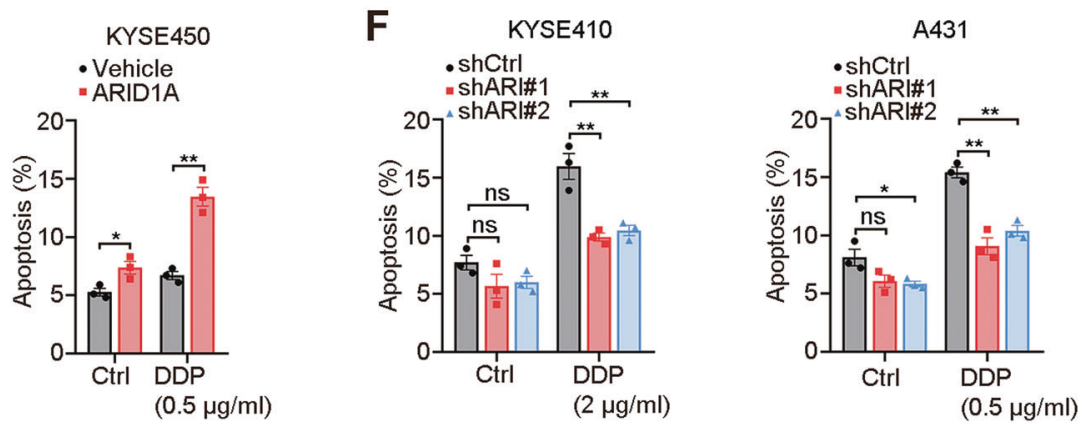
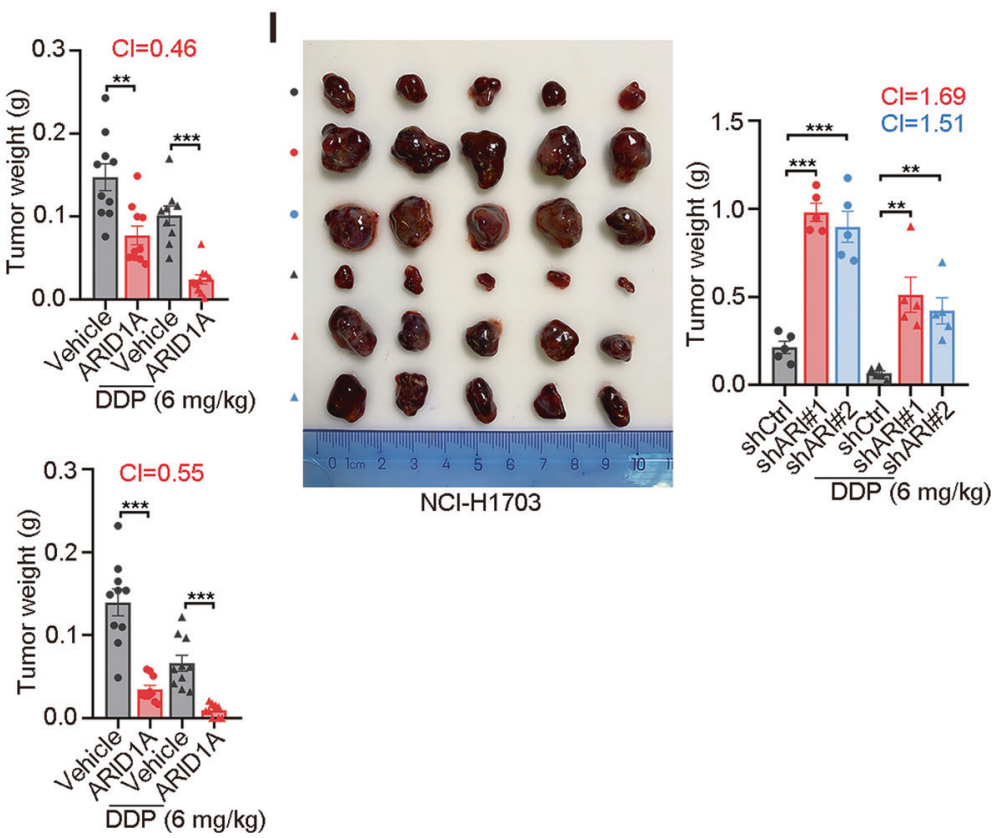

the relationship between ARID1A and E2F1, we first detected the phosphorylation of Rb upon ARID1A depletion or overexpression. Immunoblotting indicated that
ARID1A depletion increased Rb phosphorylation levels, while ARID1A overexpression decreased Rb phosphorylation levels (Fig. 5f, g). We next investigated whether 
Fig. 7 ARID1A depletion causes chemoresistance and can be rescued by a CDKi. a In vitro growth of KYSE150 and KYSE450 cells expressing the empty vector (Vehicle) or ARID1A. b Relative viability of KYSE150 and KYSE450 cells expressing the empty vector (Vehicle) or ARID1A following treatment with DDP. $\mathbf{c}$ In vitro growth of KYSE410 and A431 cells expressing control (shCtrl) or ARID1A shRNAs (shARI\#1, shARI\#2). d Relative viability of KYSE410 and A431 cells expressing control (shCtrl) or ARID1A shRNAs (shARI\#1, shARI\#2) following treatment with DDP. e Apoptotic fractions of KYSE150 and KYSE450 cells expressing the empty vector (Vehicle) or ARID1A following treatment with DDP in a flow cytometry analysis. f Apoptotic fractions of KYSE410 and A431 cells expressing control (shCtrl) or ARID1A shRNAs (shARI\#1, shARI\#2) following treatment with DDP in a flow cytometry analysis. Images and weights of tumors derived from KYSE450 (g) and KYSE150 (h) cells expressing the empty vector (Vehicle) or ARID1A following treatment with saline or DDP ( $n=10$ per group). CI cooperative index. i Images and weights of tumors derived from NCI-H1703 cells expressing control (shCtrl) or ARID1A shRNAs (shARI\#1, shARI\#2) $(n=5$ per group). CI cooperative index. Data are shown as the mean \pm s.e.m., except for $\mathbf{a}-\mathbf{d}$, which are shown as the mean \pm s.d.; $n=3$ independent experiments, two-tailed $t$ tests; $* P<0.05$, $* * P<0.01$, $* * * P<0.001$ and ns no statistical significance.

ARID1A suppressed the phosphorylation of Rb by affecting CDKs. Coimmunoprecipitation assays showed that ARID1A could interact with Rb (Fig. 5h). Moreover, ARID1A overexpression interfered with the association between CDK4/6 and Rb (Fig. 5i, j). To further specify the interaction between ARID1A and $\mathrm{Rb}$, we constructed truncated mutants of both ARID1A and Rb (Supplementary Fig. 2a, b). The A3 and A4 truncations are responsible for the interaction of ARID1A with Rb (Supplementary Fig. 2c). Moreover, ARID1A binds to the all three segments of $\mathrm{Rb}$, including the $\mathrm{C}$ terminus (Supplementary Fig. 2d), which is the same interactive region of $\mathrm{Rb}$ with $\mathrm{CDK} 4 / 6$ $[35,36]$. These results indicated that ARID1A prevented the association between CDKs and $\mathrm{Rb}$, resulting in decreased $\mathrm{pRb}$, and then suppressed E2F1 to transcriptionally inactivate c-Myc expression.

\section{ARID1A expression is negatively correlated with $\mathrm{pRb}$ and c-Myc expression}

To validate the relevance and clinical significance of the ARID1A/pRb/c-Myc axis in SCC, we first performed immunoblotting to detect the expression levels of ARID1A, $\mathrm{pRb}$, and $\mathrm{c}-\mathrm{Myc}$ in three human immortalized epithelial cell lines and several SCC cell lines. The results showed that ARID1A expression was negatively correlated with $\mathrm{pRb}$ and $\mathrm{c}-\mathrm{Myc}$ expression, and $\mathrm{pRb}$ expression was positively correlated with c-Myc expression (Fig. 6a). Further IHC analysis of 81 SCC samples also showed ubiquitously low$\mathrm{pRb} / \mathrm{c}-\mathrm{Myc}$ expression in samples with high-ARID1A expression (Fig. 6b) but high-pRb/c-Myc expression in samples with low ARID1A expression (Fig. 6c). Statistical analysis of the $H$-score revealed a significant correlation among ARID1A/pRb, ARID1A/c-Myc, and pRb/c-Myc (Fig. 6d-f). Moreover, low-ARID1A expression and high$\mathrm{pRb} / \mathrm{c}-\mathrm{Myc}$ expression correlated with poor prognosis in SCC patients (Fig. 6g-i), supporting the clinical significance of the ARID1A/pRb/c-Myc axis in SCC.

\section{ARID1A depletion promotes chemoresistance in SCC and could be sensitized by CDKi}

Resistance to chemotherapy is one of the main reasons for the poor prognosis of SCC patients. EMT has been recognized as a contributor to chemoresistance in recent years [37]; thus, we strongly speculate that low-ARID1A expression might also lead to chemoresistance in SCC. To validate our speculation, we first overexpressed ARID1A in KYSE150 and KYSE450 cells and performed CCK-8 cell viability assays. ARID1A overexpression significantly inhibited SCC cell proliferation and sensitized SCC cells to DDP treatment (Fig. 7a, b). On the other hand, the depletion of ARID1A in KYSE410 and A431 cells promoted the proliferation rate and increased their resistance to DDP treatment (Fig. 7c, d). To further validate the role of ARID1A in the chemoresistance of SCC cells, flow cytometry was performed using the annexin V-FITC/PI doublestaining method. ARID1A overexpression sensitized both KYSE150 and KYSE450 cells to DDP treatment, and ARID1A depletion in KYSE410 and A431 cells reduced their apoptosis fraction under DDP treatment (Fig. 7e, f and Supplementary Fig. 3). To study the role of ARID1A in chemoresistance in vivo, we xenografted control and ARID1A-overexpressing KYSE450 and KYSE150 cells subcutaneously into nude mice and administered either saline or DDP 1 week later. At the time of sacrifice, the tumors generated from ARID1A-overexpressing SCC cells weighed significantly less than tumors generated from control cells (Fig. 7g, h). Moreover, under the same treatment dose of DDP, the ARID1A-overexpressing groups showed a favorable decrease in tumor weight compared with the control groups, with a cooperative index (CI) of 0.46 in KYSE450 xenografts and 0.55 in KYSE150 xenografts (Fig. 7g, h). Consistent with these findings, ARID1A depletion in NCI-H1703 cells increased the resistance of xenografts to DDP treatment in vivo (Fig. 7i).

CDKis have been well studied in the treatment of various cancer types [38-40], especially breast cancer, and palbociclib is the first specific CDKi that has been approved for the treatment of estrogen receptor-positive breast cancers [41-43]. However, the indication of CDKi application for the treatment of SCCs remains unclear. Since the loss of ARID1A promoted the phosphorylation of $\mathrm{Rb}$ by CDKs, we next studied whether CDKis exert more potent effects on SCC cells with low-ARID1A expression. We knocked 


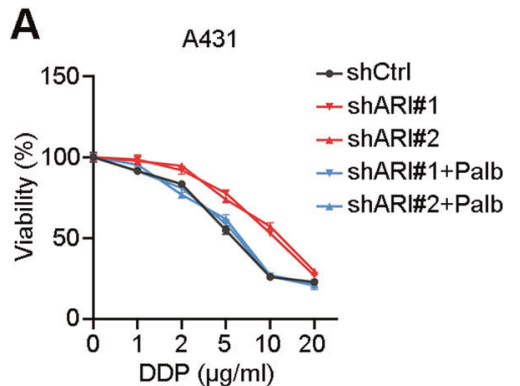

D

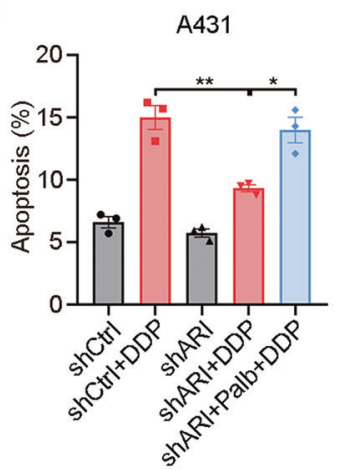

G

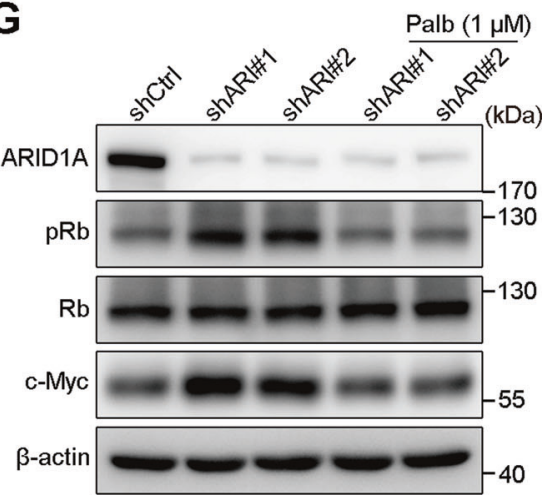

B

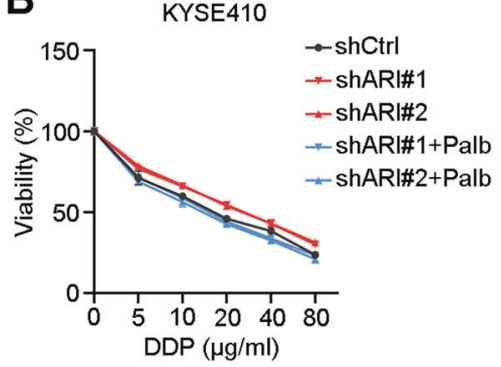

E

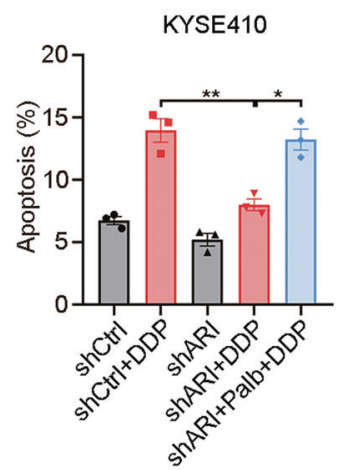

C

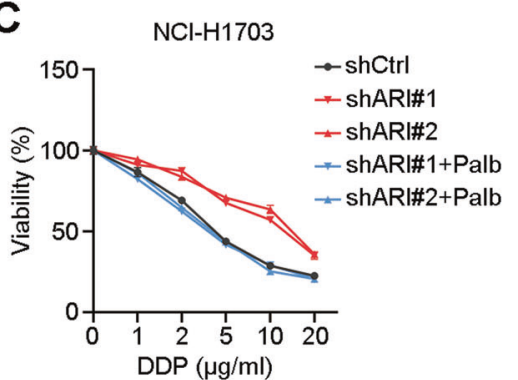

$\mathbf{F}$

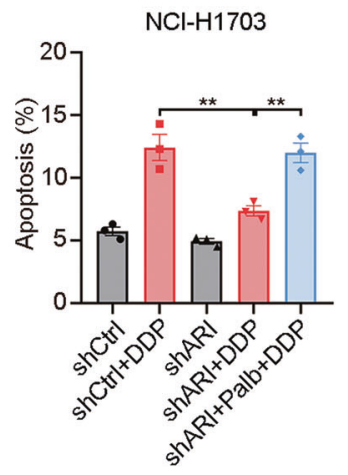

H

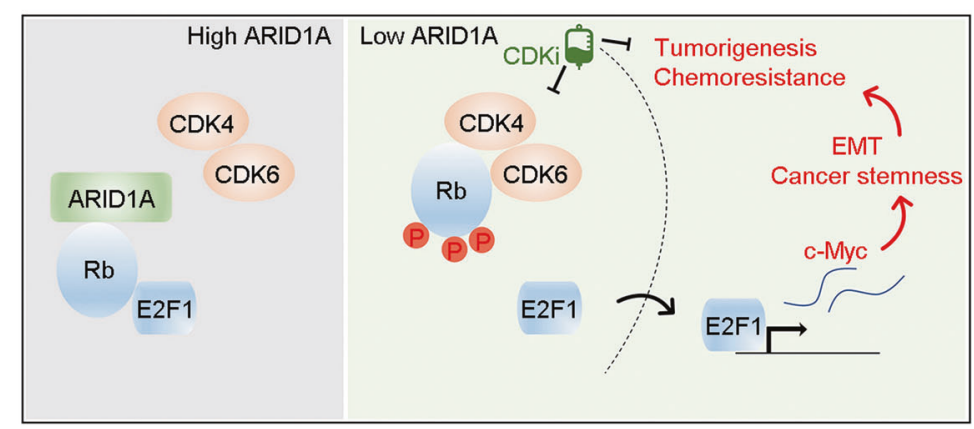

Fig. 8 CDKi sensitizes ARID1A-depleted SCC cells to DDP. a-c Relative viability of A431, KYSE410, and NCI-H1703 cells expressing control (shCtrl) or ARID1A shRNAs (shARI\#1, shARI\#2) following treatment with DDP together with or without palbociclib (1 $\mu \mathrm{M})$. d-f Apoptotic fractions of A431, KYSE410, and NCI-H1703 cells expressing control (shCtrl) or ARID1A shRNAs (shARI\#1, shARI\#2) following treatment with DDP $(0.5 \mu \mathrm{g} / \mathrm{ml}$ for A431 and NCI-H1703 cells; $2 \mu \mathrm{g} / \mathrm{ml}$ for KYSE410 cells) together with or without palbociclib $(1 \mu \mathrm{M})$ in a flow cytometry analysis. $\mathbf{g}$ Immunoblotting analysis of the indicated proteins in KYSE410 cells expressing control (shCtrl) or ARID1A shRNAs (shARI\#1, shARI\#2) following treatment with or without palbociclib $(1 \mu \mathrm{M})$. h A schematic model showing that ARID1A depletion promotes tumorigenesis and chemoresistance by increasing cancer stemness in SCC. ARID1A associates with $\mathrm{Rb}$ to prevent the association and phosphorylation of $\mathrm{Rb}$ by CDKs, while ARID1A loss promotes the phosphorylation of $\mathrm{Rb}$. Phosphorylated Rb loses its ability to suppress E2F1 transcriptional activity and then leads to the activation of c-Myc by the E2F1 transcription factor. Eventually, elevated c-Myc increases cancer stemness and contributes to tumor initiation and chemoresistance. The application of CDKi could resensitize SCC cells to DDP treatment by suppressing the $\mathrm{pRb} / \mathrm{E} 2 \mathrm{~F} 1 / \mathrm{c}-\mathrm{Myc}$ pathway. In a-c, data are shown as the mean \pm s.d.; in $\mathbf{d}-\mathbf{f}$, data are shown as the mean \pm s.e.m.; $n=3$ independent experiments, two-tailed $t$ tests; $* P<0.05$, $* * P<0.01$. down ARID1A in A431, KYSE410, and NCI-H1703 cells and then treated these cells with DDP together with or without palbociclib. CCK-8 cell viability assays showed that ARID1A depletion increased the resistance of all three SCC cell lines to DDP treatment, while the application of palbociclib significantly sensitized ARID1A-depleted SCC cells to DDP (Fig. 8a-c). Flow cytometry assays showed that ARID1A depletion led to a decreased cell apoptosis fraction compared with control cells under DDP treatment, and palbociclib treatment promoted the apoptosis of ARID1A-depleted SCC cells (Fig. 8d-f and Supplementary Fig. 4). Moreover, immunoblotting analysis showed decreased $\mathrm{pRb}$ and $\mathrm{c}-\mathrm{Myc}$ expression levels after palbociclib treatment, further supporting our speculation (Fig. 8g). In summary, our results show that ARID1A loss promotes tumorigenesis and chemoresistance in SCCs via 
increasing the phosphorylation level of Rb by CDKs and thus provide a promising strategy for applying CDKis for the treatment of SCC patients with low-ARID1A expression (Fig. 8h).

\section{Discussion}

ARID1A has become a well-known tumor suppressor in recent years, but its role in SCC is still ambiguous. Scientists have focused on ARID1A in adenocarcinomas such as ovarian cancer and gastric cancer because ARID1A shows quite high mutation rates in these cancer types. However, since mutation is only one of the causes contributing to tumor suppressor inactivation, it is still of significance to reveal the role of ARID1A in cancer types with low mutation rates, particularly SCCs. Here, we illustrate for the first time that ARID1A plays an essential tumor suppressor role in SCC. Using a conditional knockout mouse model, we showed that mice with Aridla ablation developed more severe SCC in the tongue and esophagus than wild-type mice. To further validate whether ARID1A also acts as a tumor suppressor in human SCCs, we performed both in vitro and in vivo studies and showed that ARID1A depletion promoted tumorigenesis in both human immortalized epithelial cells and SCC cells. Interestingly, the loss of ARID1A expression in the basal layer of the esophagus suggests that ARID1A depletion might contribute to tumorigenesis by increasing cancer stemness. Immunoblotting and functional studies also confirmed that ARID1A loss increased the cancer stemness and chemoresistance of SCC cells by promoting EMT.

Though many studies have revealed the tumor suppressive role of ARID1A in various cancer types, the mechanism by which ARID1A functions remains controversial. In other words, the tumor suppressor role of ARID1A might vary among different cancer types or even different contexts of one cancer type. Interestingly, a previous study also showed that ARID1A might exert an oncogenic function in liver cancer [44]. This evidence forced us to investigate the specific mechanism underlying the tumor suppressor role of ARID1A in SCC. Using RNA sequencing and GSEA, we identified c-Myc targets as the most enriched pathway in ARID1A-depleted SCC cells compared with control cells. Strikingly, a very recent study also suggested that ARID1A affects c-Myc expression, though the underlying modulatory mechanism remains unknown [45]. In subsequent studies, we showed that ARID1A competitively interacts with $\mathrm{Rb}$ and thus prevents the phosphorylation of $\mathrm{Rb}$ by CDKs. Dephosphorylated $\mathrm{Rb}$ then exerts its function to inhibit E2F1 activity. Most interestingly, we found that E2F1 acts as a transcription factor to activate c-Myc expression, in which context elevated c-Myc expression contributes to the oncogenic role of ARID1A loss in SCC. Our results elucidate the previously unknown link between ARID1A and c-Myc and reveal the essential role of the ARID1A/pRb/c-Myc axis in SCC. Due to the role of ARID1A in SWI/SNF complex-mediated chromatin remodeling, previous studies mainly focused on the direct role of ARID1A in transcriptional regulation [16, 46, 47]. In contrast, our study revealed a novel mechanism of the tumor suppressive role of ARID1A by which ARID1A functions as a blocker to interfere with the interaction between CDKs and $\mathrm{Rb}$.

EMT was previously recognized as an important event in the metastasis of cancer cells [48, 49], while studies in recent years have shown its important role in chemoresistance $[50,51]$. In this study, we showed that ARID1A loss could lead to the chemoresistance of SCC via cancer stemness caused by EMT, and the results strongly suggest that therapeutic agents that can selectively target low ARID1A-expressing SCC cells might be able to target cancer stem cells. CDKis are well-developed inhibitors that exert excellent effects against several cancer types. To date, several CDKis such as palbociclib have already been approved for clinical application in breast cancer, promoting studies to specify the favorable subtypes of their application in breast cancer [52, 53]; however, the indications of CDKis in SCCs remain undefined. In our study, palbociclib treatment rescued the increased phosphorylation level of $\mathrm{Rb}$ caused by ARID1A loss and then inhibited c-Myc expression. Functionally, palbociclib treatment resensitized ARID1A-depleted SCC cells to DDP treatment. Our study outlines a promising strategy of $\mathrm{CDKi}$ application for treating SCC patients with low-ARID1A expression, and further development of diagnostic methods based on ARID1A expression level will improve the outcomes of this strategy.

Acknowledgements We are grateful to Dr Yutaka Shimada (Kyoto University, Japan) for the ESCC cell lines and to Dr Enmin Li (Medical College of Shantou University, China) for the NE-2 and NE3 cells. We appreciate Shuhua Liu and Jingyu Zhang (Beijing Microread Genetics Co., Ltd, China) for the RNA-seq data analysis. This work was supported by the National Key R\&D Program of China (2016YFC1302100), the CAMS Innovation Fund for Medical Sciences (2016-I2M-1-001, 2019-I2M-1-003) and the National Science Foundation of China (81972570).

\section{Compliance with ethical standards}

Conflict of interest The authors declare that they have no conflict of interest.

Publisher's note Springer Nature remains neutral with regard to jurisdictional claims in published maps and institutional affiliations. 


\section{References}

1. Dotto GP, Rustgi AK. Squamous cell cancers: a unified perspective on biology and genetics. Cancer Cell. 2016;29:622-37.

2. Sanchez-Danes A, Blanpain C. Deciphering the cells of origin of squamous cell carcinomas. Nat Rev Cancer. 2018;18:549-61.

3. Alam M, Ratner D. Cutaneous squamous-cell carcinoma. N Engl J Med. 2001;344:975-83.

4. Liu J, Wang T, Creighton CJ, Wu SP, Ray M, Janardhan KS, et al. $\mathrm{JNK}(1 / 2)$ represses $\mathrm{Lkb}(1)$-deficiency-induced lung squamous cell carcinoma progression. Nat Commun. 2019;10:2148.

5. Ferlay J, Soerjomataram I, Dikshit R, Eser S, Mathers C, Rebelo $\mathrm{M}$, et al. Cancer incidence and mortality worldwide: sources, methods and major patterns in GLOBOCAN 2012. Int J Cancer. 2015;136:E359-386.

6. Pennathur A, Gibson MK, Jobe BA, Luketich JD. Oesophageal carcinoma. Lancet. 2013;381:400-12.

7. Enzinger PC, Mayer RJ. Esophageal cancer. N Engl J Med. 2003;349:2241-52.

8. Schweigert M, Dubecz A, Stein HJ. Oesophageal cancer-an overview. Nat Rev Gastroenterol Hepatol. 2013;10:230-44.

9. Demeester SR. Reoperative chemoradiotherapy for oesophageal cancer: a systematic review and meta-analysis. Gut. 2005;54: $440-1$.

10. Tsai CW, Lai FJ, Sheu HM, Lin YS, Chang TH, Jan MS, et al. WWOX suppresses autophagy for inducing apoptosis in methotrexate-treated human squamous cell carcinoma. Cell Death Dis. 2013;4:e792.

11. Bitler BG, Wu S, Park PH, Hai Y, Aird KM, Wang Y, et al. ARID1A-mutated ovarian cancers depend on HDAC6 activity. Nat Cell Biol. 2017;19:962-73.

12. Wu S, Fatkhutdinov N, Fukumoto T, Bitler BG, Park PH, Kossenkov AV, et al. SWI/SNF catalytic subunits' switch drives resistance to EZH2 inhibitors in ARID1A-mutated cells. Nat Commun. 2018;9:4116.

13. Yang F, Xu Y, Liu C, Ma C, Zou S, Xu X, et al. NF-kappaB/miR223-3p/ARID1A axis is involved in Helicobacter pylori CagAinduced gastric carcinogenesis and progression. Cell Death Dis. 2018;9:12.

14. Wang K, Kan J, Yuen ST, Shi ST, Chu KM, Law S, et al. Exome sequencing identifies frequent mutation of ARID1A in molecular subtypes of gastric cancer. Nat Genet. 2011;43:1219-23.

15. Wu C, Lyu J, Yang EJ, Liu Y, Zhang B, Shim JS. Targeting AURKA-CDC25C axis to induce synthetic lethality in ARID1A-deficient colorectal cancer cells. Nat Commun. 2018; 9:3212.

16. Mathur R, Alver BH, San Roman AK, Wilson BG, Wang X, Agoston AT, et al. ARID1A loss impairs enhancer-mediated gene regulation and drives colon cancer in mice. Nat Genet. 2017;49:296-302.

17. Williamson CT, Miller R, Pemberton HN, Jones SE, Campbell J, Konde A, et al. ATR inhibitors as a synthetic lethal therapy for tumours deficient in ARID1A. Nat Commun. 2016;7:13837.

18. Bitler BG, Aird KM, Garipov A, Li H, Amatangelo M, Kossenkov $\mathrm{AV}$, et al. Synthetic lethality by targeting EZH2 methyltransferase activity in ARID1A-mutated cancers. Nat Med. 2015;21:231-8.

19. Berns K, Caumanns JJ, Hijmans EM, Gennissen AMC, Severson TM, Evers B, et al. ARID1A mutation sensitizes most ovarian clear cell carcinomas to BET inhibitors. Oncogene. 2018;37:4611-25.

20. Shimada $Y$, Imamura $M$, Wagata $T$, Yamaguchi $N$, Tobe $T$. Characterization of 21 newly established esophageal cancer cell lines. Cancer. 1992;69:277-84.

21. Zhang H, Jin Y, Chen X, Jin C, Law S, Tsao SW, et al. Cytogenetic aberrations in immortalization of esophageal epithelial cells. Cancer Genet Cytogenet. 2006;165:25-35.
22. Luo Q, Wu X, Zhang Y, Shu T, Ding F, Chen H, et al. ARID1A ablation leads to multiple drug resistance in ovarian cancer via transcriptional activation of MRP2. Cancer Lett. 2018;427:9-17.

23. Wu X, Luo Q, Zhao P, Chang W, Wang Y, Shu T, et al. JOSD1 inhibits mitochondrial apoptotic signalling to drive acquired chemoresistance in gynaecological cancer by stabilizing MCL1. Cell Death Differ. 2019. https://doi.org/10.1038/s41418-019-0339-0.

24. Tetreault MP, Yang Y, Travis J, Yu QC, Klein-Szanto A, Tobias JW, et al. Esophageal squamous cell dysplasia and delayed differentiation with deletion of kruppel-like factor 4 in murine esophagus. Gastroenterology. 2010;139:171-81.

25. Subramanian A, Tamayo P, Mootha VK, Mukherjee S, Ebert BL, Gillette MA, et al. Gene set enrichment analysis: a knowledgebased approach for interpreting genome-wide expression profiles. Proc Natl Acad Sci USA. 2005;102:15545-50.

26. Gao X, Tate P, Hu P, Tjian R, Skarnes WC, Wang Z. ES cell pluripotency and germ-layer formation require the SWI/SNF chromatin remodeling component BAF250a. Proc Natl Acad Sci USA. 2008;105:6656-61.

27. Natsuizaka M, Whelan KA, Kagawa S, Tanaka K, Giroux V, Chandramouleeswaran PM, et al. Interplay between Notch1 and Notch3 promotes EMT and tumor initiation in squamous cell carcinoma. Nat Commun. 2017;8:1758.

28. Liu N, Matsumura H, Kato T, Ichinose S, Takada A, Namiki T, et al. Stem cell competition orchestrates skin homeostasis and ageing. Nature. 2019;568:344-50.

29. Ming XY, Fu L, Zhang LY, Qin YR, Cao TT, Chan KW, et al. Integrin alpha7 is a functional cancer stem cell surface marker in oesophageal squamous cell carcinoma. Nat Commun. 2016;7:13568.

30. Yeh HW, Hsu EC, Lee SS, Lang YD, Lin YC, Chang CY, et al. PSPC1 mediates TGF-beta1 autocrine signalling and Smad2/3 target switching to promote EMT, stemness and metastasis. Nat Cell Biol. 2018;20:479-91.

31. Muhammad N, Bhattacharya S, Steele R, Phillips N, Ray RB. Involvement of c-Fos in the promotion of cancer stem-like cell properties in head and neck squamous cell carcinoma. Clin Cancer Res. 2017;23:3120-8.

32. Khan A, Fornes O, Stigliani A, Gheorghe M, Castro-Mondragon JA, van der Lee R, et al. JASPAR 2018: update of the open-access database of transcription factor binding profiles and its web framework. Nucleic Acids Res. 2018;46:D1284.

33. Dick FA, Rubin SM. Molecular mechanisms underlying RB protein function. Nat Rev Mol Cell Biol. 2013;14:297-306.

34. Dyson NJ. RB1: a prototype tumor suppressor and an enigma. Genes Dev. 2016;30:1492-502.

35. Matsuura I, Denissova NG, Wang G, He D, Long J, Liu F. Cyclindependent kinases regulate the antiproliferative function of Smads. Nature. 2004;430:226-31.

36. Jiang W, Wells NJ, Hunter T. Multistep regulation of DNA replication by Cdk phosphorylation of HsCdc6. Proc Natl Acad Sci USA. 1999;96:6193-8.

37. Nieto MA, Huang RY, Jackson RA, Thiery JP. Emt: 2016. Cell. 2016;166:21-45.

38. Lopez-Mejia IC, Lagarrigue S, Giralt A, Martinez-Carreres L, Zanou N, Denechaud PD, et al. CDK4 phosphorylates AMPKalpha2 to inhibit its activity and repress fatty acid oxidation. Mol Cell. 2017;68:336-49.

39. Hydbring P, Wang Y, Fassl A, Li X, Matia V, Otto T, et al. Cellcycle-targeting microRNAs as therapeutic tools against refractory cancers. Cancer Cell. 2017;31:576-90.

40. Goel S, DeCristo MJ, Watt AC, BrinJones H, Sceneay J, Li BB, et al. CDK4/6 inhibition triggers anti-tumour immunity. Nature. 2017;548:471-5.

41. Fry DW, Harvey PJ, Keller PR, Elliott WL, Meade M, Trachet E, et al. Specific inhibition of cyclin-dependent kinase $4 / 6$ by PD 
0332991 and associated antitumor activity in human tumor xenografts. Mol Cancer Ther. 2004;3:1427-38.

42. Asghar U, Witkiewicz AK, Turner NC, Knudsen ES. The history and future of targeting cyclin-dependent kinases in cancer therapy. Nat Rev Drug Discov. 2015;14:130-46.

43. Finn RS, Crown JP, Lang I, Boer K, Bondarenko IM, Kulyk SO, et al. The cyclin-dependent kinase $4 / 6$ inhibitor palbociclib in combination with letrozole versus letrozole alone as first-line treatment of oestrogen receptor-positive, HER2-negative, advanced breast cancer (PALOMA-1/TRIO-18): a randomised phase 2 study. Lancet Oncol. 2015;16:25-35.

44. Sun X, Wang SC, Wei Y, Luo X, Jia Y, Li L, et al. Arid1a has context-dependent oncogenic and tumor suppressor functions in liver cancer. Cancer Cell. 2017;32:574-89.

45. Wang SC, Nassour I, Xiao S, Zhang S, Luo X, Lee J, et al. SWI/ SNF component ARID1A restrains pancreatic neoplasia formation. Gut. 2019;68:1259-70.

46. Guo X, Zhang Y, Mayakonda A, Madan V, Ding LW, Lin LH, et al. ARID1A and CEBPalpha cooperatively inhibit UCA1 transcription in breast cancer. Oncogene. 2018;37:5939-51.

47. Wilson MR, Reske JJ, Holladay J, Wilber GE, Rhodes M, Koeman J, et al. ARID1A and PI3-kinase pathway mutations in the endometrium drive epithelial transdifferentiation and collective invasion. Nat Commun. 2019;10:3554.

48. Turley EA, Veiseh M, Radisky DC, Bissell MJ. Mechanisms of disease: epithelial-mesenchymal transition-does cellular plasticity fuel neoplastic progression? Nat Clin Pract Oncol. 2008;5:280-90.

49. Brabletz T. To differentiate or not-routes towards metastasis. Nat Rev Cancer. 2012;12:425-36.

50. Fischer KR, Durrans A, Lee S, Sheng J, Li F, Wong ST, et al. Epithelial-to-mesenchymal transition is not required for lung metastasis but contributes to chemoresistance. Nature. 2015; 527:472-6.

51. Dongre A, Weinberg RA. New insights into the mechanisms of epithelial-mesenchymal transition and implications for cancer. Nat Rev Mol Cell Biol. 2019;20:69-84.

52. Drago JZ, Formisano L, Juric D, Niemierko A, Servetto A, Wander SA, et al. FGFR1 gene amplification mediates endocrine resistance but retains TORC sensitivity in metastatic hormone receptor positive $(\mathrm{HR}+)$ breast cancer. Clin Cancer Res. 2019;25:6443-51.

53. Kwapisz D. Cyclin-dependent kinase $4 / 6$ inhibitors in breast cancer: palbociclib, ribociclib, and abemaciclib. Breast Cancer Res Treat. 2017;166:41-54. 